\title{
Sampling Site Has a Critical Impact on Physiologically Based Pharmacokinetic Modeling ${ }^{\mathbf{S}}$
}

\author{
Weize Huang and (D)Nina Isoherranen \\ Department of Pharmaceutics, School of Pharmacy, University of Washington, Seattle, Washington \\ Received August 17, 2019; accepted October 9, 2019
}

\section{ABSTRACT}

It has been shown that arterial (central) and venous (peripheral) plasma drug concentrations can be very different. While pharmacokinetic studies typically measure drug concentrations from the peripheral vein such as the arm vein, physiologically based pharmacokinetic (PBPK) models generally output simulated concentrations from the central venous compartment that physiologically represents the right atrium, a merge of the superior and inferior vena cava. In this study, a physiologically based peripheral forearm sampling site model was developed and verified using nicotine, ketamine, lidocaine, and fentanyl as model drugs. This verified model allows output of simulated peripheral venous concentrations that can be meaningfully compared with observed pharmacokinetic data from the arm vein. The generalized effect of PBPK model sampling site on simulation output was investigated. Drugs and metabolites with large volumes of distribution showed considerable concentration discrepancy between the simulated central venous compartment and the peripheral arm vein after intravenous or oral administration, resulting in significant differences in values for $C_{\max }$ and time taken to reach $C_{\max }\left(t_{\max }\right)$ In addition, the simulated central venous metabolite profile showed an unexpected profile that was not observed in the peripheral arm vein. Using fentanyl as a model compound, we show that using the wrong sampling site in PBPK models can lead to biased model evaluation and subsequent erroneous model parameter optimization. Such an error in model parameters along with the discrepant sampling site could dramatically mislead the pharmacokinetic prediction in unstudied clinical scenarios, affecting the assessment of drug safety and efficacy. Overall, this study shows that PBPK model publications should specify the model sampling sites and match them with those employed in clinical studies.

\section{SIGNIFICANCE STATEMENT}

Our study shows that sampling from the central venous compartment (right atrium) during physiologically based pharmacokinetic model development gives rise to biased model evaluation and erroneous model parameterization when observed data are collected from the peripheral arm vein. This can lead to a clinically significant error in predictions of plasma concentration-time profiles in unstudied scenarios. To address this error, we developed and verified a novel peripheral sampling site model to simulate arm vein drug concentrations that can be applied to different drug dosing scenarios.

\section{Introduction}

Physiologically based pharmacokinetic (PBPK) modeling has been widely used to facilitate design of first-in-human dosing (Jones et al., 2013; Offman and Edginton, 2015; Miller et al., 2019), estimate magnitude of drug-drug interactions (Min and Bae, 2017; Britz et al., 2019; Grimstein et al., 2019), predict pharmacokinetic (PK) parameters (Huang and Isoherranen, 2018; Kaur et al., 2018), and simulate drug disposition in specific populations (Khalil and Läer, 2011; Rowland Yeo et al., 2011b; Huang et al., 2017). The underlying theory (Jones et al., 2006; Rowland et al., 2011a; Jones and RowlandYeo, 2013) and common applications (Jones et al., 2012; Sager et al., 2015; Jamei, 2016) of PBPK models have been extensively

This work was supported by the National Institutes of Health National Institute on Drug Abuse [Grant P01-DA032507]. W.H. was supported by a Warren G. Magnuson Scholarship and a William E. Bradley Fellowship from the University of Washington.

https://doi.org/10.1124/jpet.119.262154.

S This article has supplemental material available at jpet.aspetjournals.org. reviewed in the literature. Regulatory agencies also use PBPK modeling to facilitate decision making during the drug approval process (Huang et al., 2013; Luzon et al., 2017; Shebley et al., 2018). Surprisingly, the sampling site for drug concentration data, the primary PBPK simulation output, is rarely explicitly discussed. Simulation output is typically the drug concentration in the central venous compartment that drains blood from the tissue/organ compartments (Kuepfer et al., 2016). This central venous compartment is built in the PBPK models as a mixed compartment that merges all of the tissue/organ-specific veins. Physiologically, this central venous compartment resembles the right atrium where the superior and inferior vena cava merge together. However, in clinical practice and in PK studies, plasma samples (i.e., the observed data) are usually taken from a peripheral sampling site such as the median cubital vein (i.e., arm vein). This generates a concerning discrepancy between the modeled and observed data because measured drug concentrations from the arm artery, which are essentially equivalent to the drug concentrations in the right atrium (assuming lung distribution is not significant and distribution equilibrium in the

ABBREVIATIONS: AAFE, absolute average fold error; AUC, area under the plasma drug concentration-versus-time curve; CL, total plasma

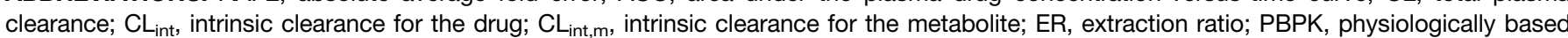
pharmacokinetics; PD, pharmacodynamics; PK, pharmacokinetics; $t_{\max }$, time taken to reach $C_{\max } ; V_{\mathrm{ss}}$, volume of distribution at steady state. 
lung is reached instantaneously), have been shown to differ from concentrations in the peripheral vein for multiple drugs (Chiou, 1989; Gourlay and Benowitz, 1997; Ericsson et al., 2000; Rentsch et al., 2001; Persson et al., 2002; Darwish et al., 2006a; Olofsen et al., 2010). This difference in arteriovenous drug concentrations has been shown to affect pharmacokinetic-pharmacodynamic (PK-PD) modeling when the PD effect is empirically fitted to plasma drug concentrations (Gumbleton et al., 1994; Jacobs and Nath, 1995; Tuk et al., 1997). As a result of the observed discrepancy between arterial and arm vein concentrations, the derived PK-PD relationship using different concentrations as a reference may differ. This may result in misspecified PK-PD models that fail to predict PD in other circumstances. Based on the observed arteriovenous drug concentration differences, we hypothesized that PBPK model-simulated concentration profiles, model parameterization, model acceptance, and model extrapolation will be impacted by model sampling site.

Two previous studies that developed a virtual peripheral blood compartment to model drug concentrations at the peripheral sampling site showed differences in the simulated concentrations depending on the sampling site (Levitt, 2004; Musther et al., 2015). However, neither study established a physiologically based forearm compartment, which prevents capturing the unique forearm tissue distribution phenomenon at the sampling site. Instead, both studies employed an empirical fitting strategy to estimate peripheral blood concentration. The first study (Levitt, 2004) mentioned that such fitting could not be conducted in a global manner and the verification of the fitted peripheral site using a different set of test drugs was unsuccessful. The second study (Musther et al., 2015) modified Levitt's model but did not verify the model using arterial concentrations or arteriovenous difference. In addition, neither study addressed: 1) the general impact of sampling site on different compounds with varying properties, 2) the arteriovenous difference after oral dosing, 3) the arteriovenous difference for drug metabolites, and 4) the impact of model sampling site selection on PBPK model development, evaluation, and application. Hence, further studies are needed to understand the importance of sampling site selection in PBPK modeling. In this study, a physiologically based forearm compartment was developed and verified as the peripheral sampling site in the PBPK model. The verified model was then used to explore the differences in the simulated concentrations between different PBPK model sampling sites after intravenous and oral dosing for drugs and metabolites with a wide spectrum of PK properties. Finally, the model was applied to simulate central arterial and peripheral arm vein concentrations after fentanyl buccal administration to demonstrate the impact of discrepant sampling site on model evaluation, parameter optimization, and prediction in unstudied scenarios during model application and extrapolation.

\section{Materials and Methods}

Development of a Full-Body PBPK Parent-Metabolite Structural Model with a Peripheral Forearm Sampling Site. A 32-compartment parent-metabolite full-body PBPK model (Fig. 1) was developed using MATLAB and the Simulink platform (R2018a; MathWorks, Natick, MA). A central venous (i.e., physiologically analogous to the right atrium) compartment was included to merge all organ/tissue-specific emergent veins before encountering the lung compartment. A central arterial compartment was built to receive blood from the lungs and distribute blood flows to all tissue compartments. Similar to conventional full PBPK models (Rowland et al., 2011a; Jones and Rowland-Yeo, 2013), 12 physiologically important tissues and organs (adipose, bone, brain, gut, heart, kidney, liver, lung, muscle, skin, spleen, and pancreas) were included in the model (Supplemental Table 1). Except the liver, all other tissue/organ compartments including the forearm were modeled as perfusion-limited compartments where drug distribution was assumed to reach equilibrium instantaneously between tissue mass and tissue blood, with a total drug concentration gradient defined by the tissue-to-plasma partition coefficient $\left(K_{\mathrm{p}}\right)$. For the liver, a permeability-limited model including two compartments, the hepatocytes and the sinusoidal blood, was built. In this study, the exchange of drug between the two compartments was determined by passive diffusion, which was set to be sufficiently high to reflect perfusion-limited hepatic distribution. The hepatic metabolism of drug and metabolite and the formation of metabolite in the liver were modeled to occur within the hepatocyte compartment (Fig. 1).

A forearm compartment was incorporated in parallel with other distribution organs based on known physiology to capture drug distribution to the peripheral sampling site (Fig. 1). Since the median cubital vein is a superficial vein that mostly drains from adipose, muscle, and skin tissues of the forearm, the forearm compartment only included these three tissue types and neglected bone, which is believed to be drained only by the deep vein. The model parameters of volumes (Cooper et al., 1955) and blood flows (Elia and Kurpad, 1993) of the forearm adipose, muscle, and skin are physiologic values in humans and are summarized in Supplemental Table 1. Each of the forearm tissues was assumed to share the same $K_{\mathrm{p}}$ value as the respective tissues in the rest of the body. Structurally, the forearm compartment was modeled as a perfusion-limited tissue with anastomoses taken into account as a blood flow shunt directly from the arm artery into the arm vein. A previously applied fit-for-purpose anastomoses fraction of $10 \%$ (Musther et al., 2015) was used in the arm model in this study. Sensitivity analyses were conducted (Supplemental Figs. 1 and 2) to examine the potential impact of parameter uncertainty on central venous compartment (right atrium) and peripheral arm vein drug concentrations. Specifically, the influence of $K_{\mathrm{p}}$ values of muscle, adipose, and skin tissues and anastomoses fraction were tested. For the sensitivity analysis, the baseline condition was set with $K_{\mathrm{p}}$ values for all tissues equal to 3 [volume of distribution at steady state $\left(\mathrm{V}_{\mathrm{ss}}\right)=$ 200 liters] and anastomoses fraction equal to 10\%. Overall, two sets of scenarios were tested after intravenous administration. For the first set of scenarios, $K_{\mathrm{p}}$ values of muscle, adipose, or skin were either increased from 3 to 5 or decreased from 3 to 1 . In all scenarios, $K_{\mathrm{p}}$ values for other untested tissues were adjusted uniformly such that the $\mathrm{V}_{\mathrm{ss}}$ remained at 200 liters to avoid the impact of total volume changes on drug disposition and the simulated plasma concentration curves. In addition, the impact of the anastomoses fraction was examined by increasing the anastomoses fraction from $10 \%$ to $30 \%$ and decreasing it from $10 \%$ to $0 \%$. For the second set of scenarios, $K_{\mathrm{p}}$ values of muscle, adipose, or skin were either increased from 3 to 10 or decreased from 3 to 1 without controlling for $\mathrm{V}_{\mathrm{ss}}$, so that only one parameter was changed at a time and a wider range of $K_{\mathrm{p}}$ values could be tested. For sampling in the simulations, plasma drug concentrations were sampled from the central venous compartment (right atrium), central artery compartment, and peripheral forearm vein (Fig. 1). The model file and code script are provided in the Supplemental Material.

Verification of the Peripheral Forearm Sampling Site Model. To verify our developed physiologically based forearm model as the peripheral sampling site, six observed PK studies conducted with nicotine, ketamine, lidocaine, and fentanyl after intravenous administration were used (Tucker and Boas, 1971; Gourlay and Benowitz, 1997; Persson et al., 2002; Isohanni et al., 2005; Macleod et al., 2012; Ziesenitz et al., 2015). Plasma concentration-time curves from these studies were digitized using WebPlotDigitizer (version 4.2, https:// automeris.io/WebPlotDigitizer). To simulate the plasma concentrationtime profile for nicotine, ketamine, lidocaine, and fentanyl, drug-specific models for individual compounds were built and detailed drug 
parameters are shown in Table 1. The physicochemical properties, plasma unbound fraction, and blood-to-plasma ratios were collected from literature for each compound (Rodgers and Rowland, 2007). Tissue-specific $K_{\mathrm{p}}$ values that have been experimentally determined in the rat were used for each drug (Edwards and Mather, 2001; Rodgers et al., 2005). For the tissues/organs that did not have experimentally determined $K_{\mathrm{p}}$ data for a given drug, a value of 1 was used. The systemic plasma clearances that were reported in each of the corresponding human studies used for verification were used in the model (Persson et al., 2002; Pitsiu et al., 2002; Isohanni et al., 2005; Macleod et al., 2012; Ziesenitz et al., 2015). One study (Tucker and Boas, 1971) did not report the observed clearance value; therefore, a secondary source (Goodman et al., 2006) was used for lidocaine clearance. The hepatic intrinsic clearance was calculated assuming a well stirred model and the systemic clearances were all attributed to hepatic clearance. All simulations used the same dosage,

A

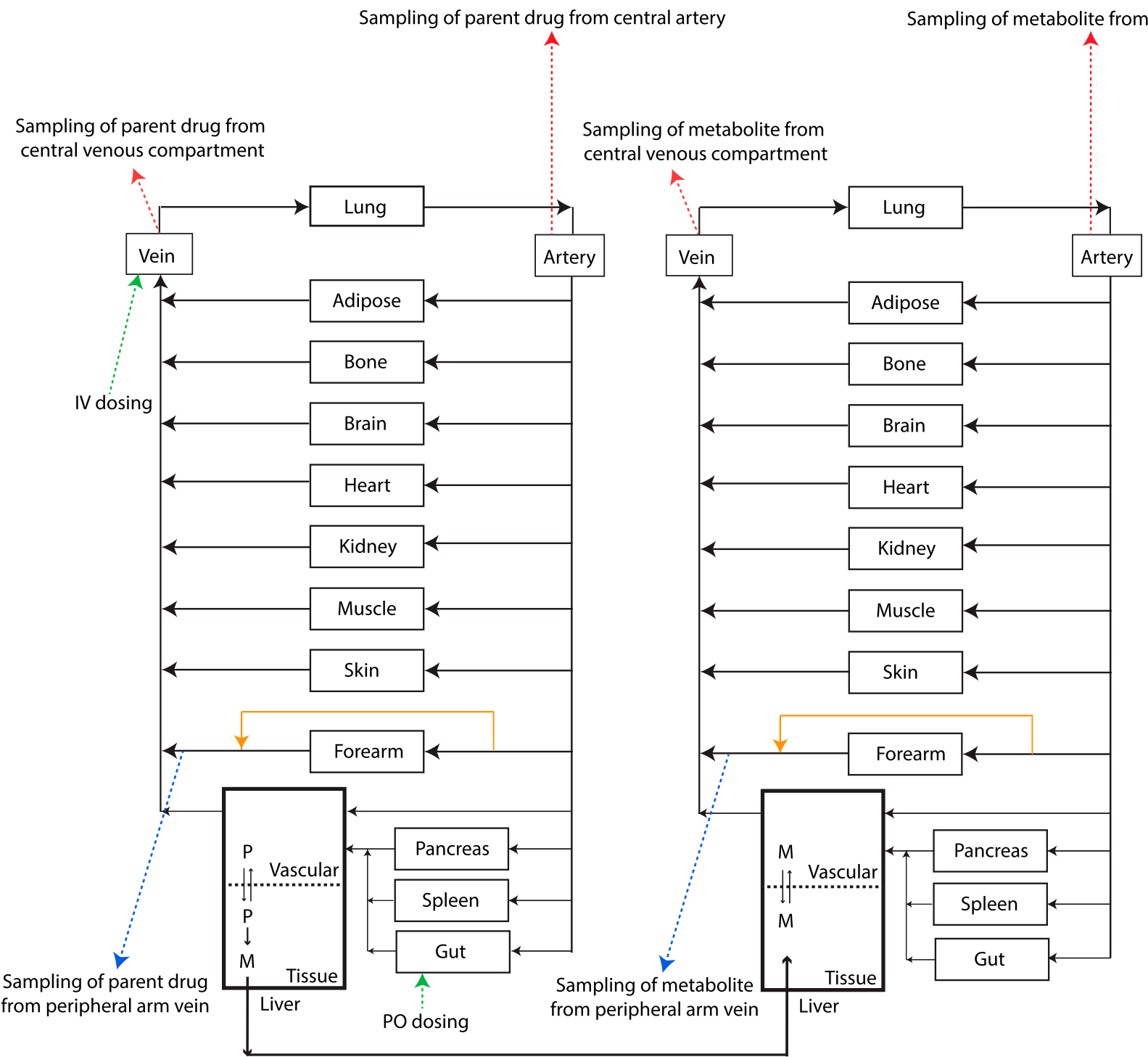

B

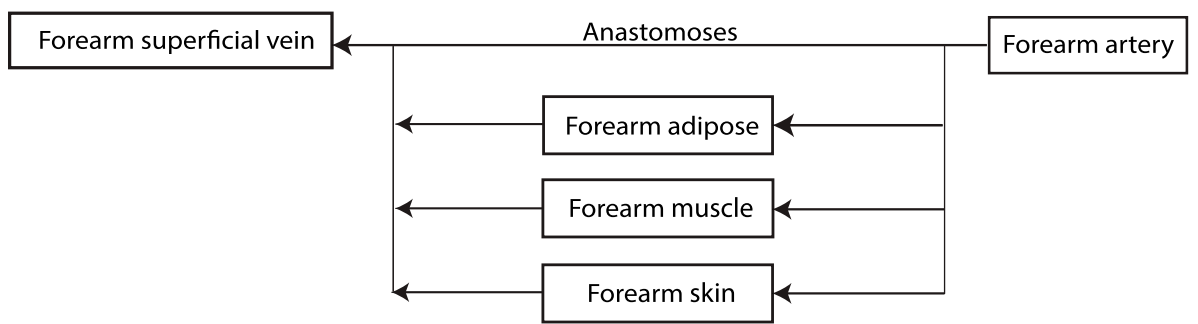

Fig. 1. Structure of the PBPK model and the forearm sampling site developed. (A) Schematic diagram of the full-body physiologically based parent (P)metabolite (M) PK model with the forearm compartment incorporated. Three different sampling sites of drug and metabolite are shown. Sampling from the central venous compartment (right atrium) and the central artery compartment are shown with red dashed lines and sampling from the peripheral arm vein is shown with blue dashed lines. The intravenous (IV) and oral (PO) dosing sites are shown with green dashed lines. The forearm anastomoses shunt is shown by orange line. (B) The detailed structural model of the forearm compartment. 
TABLE 1

Physicochemical and PK parameters of drugs used for structural model verification

\begin{tabular}{|c|c|c|c|c|}
\hline Parameter & Nicotine ${ }^{a}$ & Ketamine $^{b}$ & Lidocaine $^{a}$ & Fentanyl $^{a}$ \\
\hline \multicolumn{5}{|l|}{ Physicochemical $^{c}$} \\
\hline Molecular weight (g/mol) & 162.23 & 237.73 & 234.34 & 336.47 \\
\hline $\mathrm{p} K_{\mathrm{a}}$ & 7.80 & 7.50 & 8.01 & 8.99 \\
\hline$f_{u, p}$ & 0.84 & 0.77 & 0.35 & 0.16 \\
\hline $\mathrm{B} / \mathrm{P}$ & 0.80 & 1.24 & 0.87 & 1.00 \\
\hline \multicolumn{5}{|l|}{ Distribution } \\
\hline$K_{\text {p,adipose }}$ & 0.50 & 2.8 & 1.0 & 26.7 \\
\hline$K_{\mathrm{p}, \text { gastrointestinal tract }}$ & 1.0 & 5.8 & 3.1 & 8.4 \\
\hline$K_{\text {p,heart }}$ & 1.9 & 6.8 & 2.7 & 4.5 \\
\hline$K_{\mathrm{p}, \text { kidney }}$ & 13.3 & 11.3 & 17.2 & 12.1 \\
\hline$K_{\mathrm{p}, \text { liver }}$ & 3.5 & 5.3 & 11.5 & 3.8 \\
\hline$K_{\mathrm{p}, \text { lung }}$ & 2.1 & 7.6 & 3.8 & 13.5 \\
\hline$K_{\mathrm{p}, \text { muscle }}$ & 1.6 & 2.0 & 1.7 & 3.1 \\
\hline$K_{\mathrm{p}, \text { pancreas }}$ & 1.0 & 1.0 & 1.0 & 21.3 \\
\hline
\end{tabular}

$\mathrm{B} / \mathrm{P}$, blood-to-plasma ratio; $\mathrm{CL}_{\mathrm{total}}$, observed clearance for each study; $\mathrm{f}_{\mathrm{u}, \mathrm{p}}$, fraction unbound in plasma; $K_{\mathrm{p}}$, tissue-to-plasma partition coefficient for specific organ/tissue.

${ }^{a} K_{\mathrm{p}}$ data collected from Rodgers et al. (2005).

${ }^{b} K_{\mathrm{p}}$ data collected from Edwards and Mather (2001).

${ }^{c}$ Physicochemical data collected from Rodgers and Rowland (2007).

${ }^{d}$ Letters in parentheses indicate the following: (a), clinical studies used for model verification that report arterial data; (b), a clinical study that uses buccal administration; and (v), clinical studies used for model verification that report venous data.

route of administration, and infusion length as described in the original clinical studies (Tucker and Boas, 1971; Gourlay and Benowitz, 1997; Persson et al., 2002; Isohanni et al., 2005; Macleod et al., 2012; Ziesenitz et al., 2015). A representative virtual subject was used in all simulations with typical physiologic values without variability. The simulated plasma drug concentrations were sampled from the central venous compartment (right atrium), which is essentially equal to the arterial compartment, and from the developed peripheral arm vein compartment for comparison with the observed arterial and arm vein concentrations, respectively.

For quantitative evaluation and model verification, the absolute average fold error (AAFE; eq. 1) between the observed and simulated data was calculated. The model was considered acceptable if the AAFE was within the 1.5 -fold criteria.

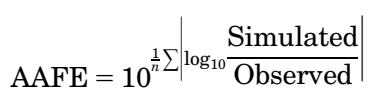

Sampling Site Effect on PBPK Model-Simulated Plasma Drug Concentrations after Intravenous Administration. The verified model was used to investigate whether the effect of PBPK model sampling site on simulated plasma drug concentration-time profiles and PK parameters could be generalized for a wide range of compounds that have not been experimentally studied for arteriovenous drug concentration difference. A matrix of nine hypothetical drugs with a range of clearance and distribution characteristics was produced for generalization purposes. The simulated hypothetical drugs had metabolic intrinsic clearance $\left(\mathrm{CL}_{\mathrm{int}}\right)$ and extraction ratio $(\mathrm{ER})$ values that ranged from low $\left(\mathrm{CL}_{\mathrm{int}}=10 \mathrm{l} / \mathrm{hour}, \mathrm{ER}=0.1\right)$ to medium $\left(\mathrm{CL}_{\text {int }}=100 \mathrm{l} / \mathrm{hour}, \mathrm{ER}=0.53\right)$ and high $\left(\mathrm{CL}_{\text {int }}=1000 \mathrm{l} / \mathrm{hour}\right.$, $\mathrm{ER}=0.92)$ and distribution characteristics that varied from $\mathrm{a} \mathrm{V}_{\mathrm{ss}}$ of 66 liters $\left(K_{\mathrm{p}}=1\right.$ for all organs) to 189 liters $\left(K_{\mathrm{p}}=3\right.$ for all organs $)$ and 619 liters $\left(K_{\mathrm{p}}=10\right.$ for all organs). The blood-to-plasma ratio and plasma unbound fraction were 1 for all simulations and renal and biliary clearances were set as 0 . All simulations were run for 240 hours after dosing. The area under the plasma drug concentration-versus-time curves (AUCs) were calculated from $t=0-240$ hours using the trapezoidal method in MATLAB, and the $C_{\text {last }}$ value was the drug concentration at $t=240$ hours. To simulate intravenous dosing, $1000 \mathrm{mg}$ drug was infused at a constant rate into the central venous compartment (right atrium). First, intravenous infusion times of 1,5 , 10, 20, and 30 minutes were tested (Supplemental Fig. 3). Based on these simulations, a 1-minute infusion time was selected and used for all subsequent simulations of intravenous bolus dosing.

Verification of the Fentanyl Model after Buccal Administration. To explore the arteriovenous differences for route of administration other than intravenously, the fentanyl drug model verified for intravenous administration was used and expanded to capture arteriovenous differences after buccal administration. A PK study that reported both arterial and peripheral arm vein fentanyl concentrations after buccal administration was used as the training data set for model development (Darwish et al., 2006a). Plasma concentrationtime curves were digitized using WebPlotDigitizer (version 4.2, https:// automeris.io/WebPlotDigitizer). To simulate fentanyl buccal administration, $50 \%$ of the dose was absorbed through the buccal epithelium and the other $50 \%$ was absorbed through the gut based on the drug label (U.S. Food and Drug Administration, 2011). The absorption was assumed to be complete from both buccal and the gastrointestinal routes [i.e., fraction absorbed $\left(\mathrm{F}_{\mathrm{a}}\right)=1$ ]. Intestinal metabolism was assumed to be zero (i.e., $\left.\mathrm{F}_{\mathrm{g}}=1\right)$. The buccal absorption rate constant $\left(k_{\mathrm{a}, \text { buccal }}\right)$ and gut absorption rate constant $\left(k_{\mathrm{a}, \text { gut }}\right)$ were optimized to be 1 and 3 hours $^{-1}$, respectively, based on the training data set (Darwish et al., 2006a). To verify the optimized absorption rate parameters, three independent data sets (Darwish et al., 2006b, 2010) that reported only peripheral arm vein concentrations were used as the test data set. During the verification, the simulated plasma drug concentrations sampled from the developed forearm vein compartment were compared with the observed peripheral arm vein concentrations. For quantitative evaluation and model verification, the $\mathrm{AAFE}$ and the $C_{\max }$ ratio were calculated. The model was considered acceptable if the AAFE was within the conventional 2 -fold criteria and the $C_{\max }$ ratio was within the 0.8 to 1.25-fold range.

Sampling Site Effect on PBPK Model-Simulated Plasma Drug Concentrations after Oral Administration. After verification of fentanyl buccal administration, the model was used to investigate the effect of PBPK model sampling site on simulated plasma drug concentration-time profiles for a set of hypothetical drugs with 
a wide range of $\mathrm{PK}$ characteristics after oral administration. A matrix of 15 hypothetical drugs with a range of clearance and distribution parameters was generated. The simulated hypothetical drugs had clearances that ranged from low $\left(\mathrm{CL}_{\mathrm{int}}=10 \mathrm{l} / \mathrm{hour}, \mathrm{ER}=0.1\right)$ to medium $\left(\mathrm{CL}_{\text {int }}=100 \mathrm{l} /\right.$ hour, $\left.\mathrm{ER}=0.53\right)$ and high $\left(\mathrm{CL}_{\text {int }}=1000 \mathrm{l} / \mathrm{hour}\right.$, $\mathrm{ER}=0.92$ ) and distribution characteristics that varied from $\mathrm{a} \mathrm{V}_{\mathrm{ss}}$ of 66 liters $\left(K_{\mathrm{p}}=1\right.$ for all organs) to 189 liters $\left(K_{\mathrm{p}}=3\right.$ for all organs), 619 liters $\left(K_{\mathrm{p}}=10\right.$ for all organs), 209 liters $\left(K_{\mathrm{p}}=1\right.$ for adipose, muscle, and skin and $K_{\mathrm{p}}=10$ for all other organs), and 477 liters $\left(K_{\mathrm{p}}=10\right.$ for adipose, muscle, and skin and $K_{\mathrm{p}}=1$ for all other organs). The bloodto-plasma ratio and plasma unbound fraction were 1 for all simulations and renal and biliary clearances were set as 0 . All simulations were run for 240 hours after dosing, the AUCs from $t=0-240$ hours were calculated using the trapezoidal method in MATLAB, and the $C_{\text {last }}$ value was the drug concentration at $t=240$ hours. To simulate oral dosing, $1000 \mathrm{mg}$ drug was introduced into the gut lumen followed by immediate complete dissolution and homogeneous distribution inside the lumen. Drug absorption from the gut lumen into intestinal blood was described by a single first-order rate constant $k_{\mathrm{a}}$. Drug appearance in the portal vein was driven by both $k_{\mathrm{a}}$ and the blood flow of the gut. $\mathrm{F}_{\mathrm{a}}$ was assumed to be 1 and no intestinal metabolism occurred. First, $k_{\mathrm{a}}$ values of $0.2,0.5,1,2$, and 5 hours $^{-1}$ were simulated (Supplemental Fig. 4); based on these simulations, a $k_{\mathrm{a}}$ value of 1 hour $^{-1}$ was selected for the matrix of oral dosing simulations unless otherwise stated.

Sampling Site Effect on PBPK Model Evaluation, Parameter Optimization, and Model Extrapolation. To explore the potential impact of the use of a discrepant sampling site between PBPK model simulations and observed studies on model evaluation during top-down model development, fentanyl disposition after buccal administration was used as a representative scenario. Three observed data sets of fentanyl buccal administration (Darwish et al., 2006b, 2010) that reported only peripheral arm vein sampling were used and the simulated central venous compartment (right atrium) fentanyl concentrations were compared with the observed arm vein data. The model evaluations were considered acceptable if the $C_{\max }$ ratio met the 0.8- to 1.25-fold criterion and if the AAFE was within the conventional 2 -fold criterion. The $k_{\mathrm{a}}$ values in the fentanyl model were then optimized using the central venous compartment (right atrium) as the sampling site (Darwish et al., 2010) so that the simulated central venous compartment concentrations met the model verification criteria when compared with the observed arm vein data.

The collective impact of the choice of sampling site on PBPK model development and the rigor in predicting unstudied situations was evaluated in a hypothetical scenario in which a new buccal formulation of fentanyl was created with an estimated $400 \%$ increase in the buccal absorption rate. Both the arm vein model with verified $k_{\mathrm{a}}$ values and the central venous compartment sampling model with $k_{\mathrm{a}}$ values optimized using the discrepant sampling site were used to simulate fentanyl PK in this unstudied clinical scenario. The predicted $C_{\max }$ change for the new formulation was calculated based on the arm vein model and the central venous compartment sampling model.

Sampling Site Effect on PBPK Model-Simulated Plasma Metabolite Concentrations. The impact of sampling site on simulated metabolite kinetics was studied via simulations of plasma concentration-time curves of metabolites with a matrix of different drug and metabolite characteristics. Three scenarios of hypothetical drug and metabolite intrinsic clearance $\left(\mathrm{CL}_{\mathrm{int}}\right.$ and $\mathrm{CL}_{\mathrm{int}, \mathrm{m}}$, respectively) combinations were simulated: 1) $\mathrm{CL}_{\mathrm{int}}=10 \mathrm{l} / \mathrm{hour}$ and $\mathrm{CL}_{\mathrm{int}, \mathrm{m}}=20$ l/hour, 2) $\mathrm{CL}_{\mathrm{int}}=1000 \mathrm{l} /$ hour and $\mathrm{CL}_{\mathrm{int}, \mathrm{m}}=20 \mathrm{l} / \mathrm{hour}$, and 3) $\mathrm{CL}_{\mathrm{int}}=10$ 1/hour and $\mathrm{CL}_{\mathrm{int}, \mathrm{m}}=2000 \mathrm{l} / \mathrm{hour}$. For each combination of drug and metabolite intrinsic clearances, different distribution kinetics of the metabolite were simulated: 1$)$ metabolite $K_{\mathrm{p}}\left(K_{\mathrm{p}, \mathrm{m}}\right)=1$,2) $K_{\mathrm{p}, \mathrm{m}}=3$, and 3) $K_{\mathrm{p}, \mathrm{m}}=10$ for all organs, with the drug $K_{\mathrm{p}}$ values of all organs equal to 3 . For the simulations, the drug was administered as either a $1000 \mathrm{mg}$ intravenous bolus over 1 minute into the central venous compartment or a $1000 \mathrm{mg}$ oral dose into the gut lumen as described above. $k_{\mathrm{a}}$ and $\mathrm{F}_{\mathrm{a}}$ were as described above and no metabolite was formed in the gut tissue.

\section{Results}

Development and Verification of the Full-Body PBPK Structural Model with Peripheral Forearm Sampling Site. A full PBPK model with a novel peripheral forearm sampling site was developed using a conventional PBPK model structure and incorporating both peripheral and central sampling sites (Fig. 1). A forearm compartment consisting of forearm muscle, forearm skin, and forearm adipose with human physiology-based volumes and blood flows for each tissue was included in the model to allow arm vein sampling (Supplemental Table 1). To evaluate the impact of parameter uncertainty on central venous compartment (right atrium) and peripheral arm vein concentrations, sensitivity analyses were conducted. The results (Supplemental Figs. 1 and 2) show that the $K_{\mathrm{p}}$ of muscle is the most sensitive parameter, which would be expected because the forearm predominantly consists of muscle tissue and the blood perfusion rate for muscle is the lowest among all forearm tissues (Supplemental Table 1). In addition, an optimized anastomoses fraction of $10 \%$ (Musther et al., 2015) was used in this model, and the sensitivity analyses show that this parameter is not critically sensitive (Supplemental Fig. 1). The sensitivity analyses also show that although the arm vein concentration profile is sensitive to the $K_{\mathrm{p}}$ values or arm tissues and anastomoses fraction, the arterial and central venous compartment (right atrium) concentrations are relatively insensitive to these parameters.

To verify the developed model, nicotine, ketamine, lidocaine, and fentanyl PK data collected from arm artery and vein after intravenous administration were used (Fig. 2). These drugs were chosen after an extensive literature search, based on data availability of arterial and arm vein concentrations and experimentally measured $K_{\mathrm{p}}$ values in animals. PK data from six clinical studies, in which arterial or arm vein drug concentrations were reported after intravenous administration, were collected (Tucker and Boas, 1971; Gourlay and Benowitz, 1997; Persson et al., 2002; Isohanni et al., 2005; Macleod et al., 2012; Ziesenitz et al., 2015). Notably, nicotine and ketamine studies reported simultaneous arterial and arm vein drug concentration-time profiles during and after intravenous infusion. The average fold difference between the observed arterial and arm vein data across all analyzed time points was 1.7 for nicotine (Fig. 2A) and 1.6 for ketamine (Fig. 2B), illustrating the considerable differences between arterial and arm vein drug concentrations during and after intravenous infusion.

To simulate the observed PK data and to verify the developed model, drug-specific models for nicotine, ketamine, lidocaine, and fentanyl were developed (Table 1), both central venous compartment (right atrium) and peripheral arm vein concentrations were simulated, and the simulations were compared with the observed arterial and arm vein data, respectively. Overall, both arterial and arm vein concentrations were successfully recapitulated for all four drugs in all seven studies with AAFE values within 1.5-fold (Fig. 2), verifying the developed forearm structural model. The simulated drug concentrationtime profiles sampled from the central venous compartment (right atrium) superimposed with the simulated drug concentrationtime profiles sampled from the artery compartment (Fig. 2, A-C and $\mathrm{E}$ ), confirming that the central venous compartment (right atrium) 

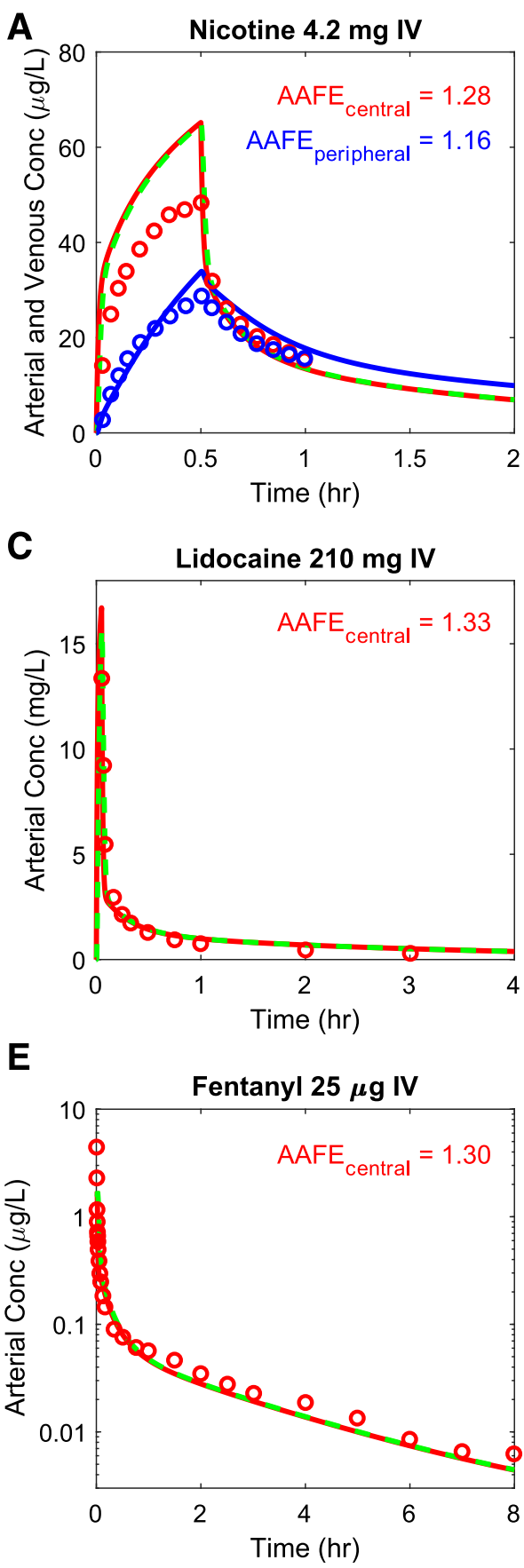
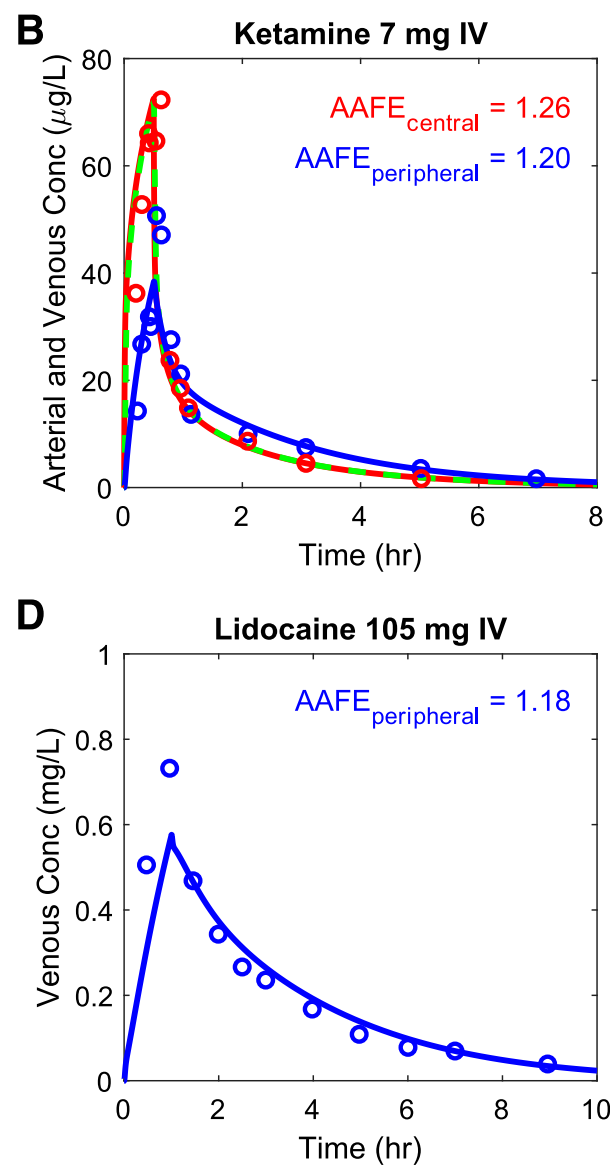

$\mathbf{F}$

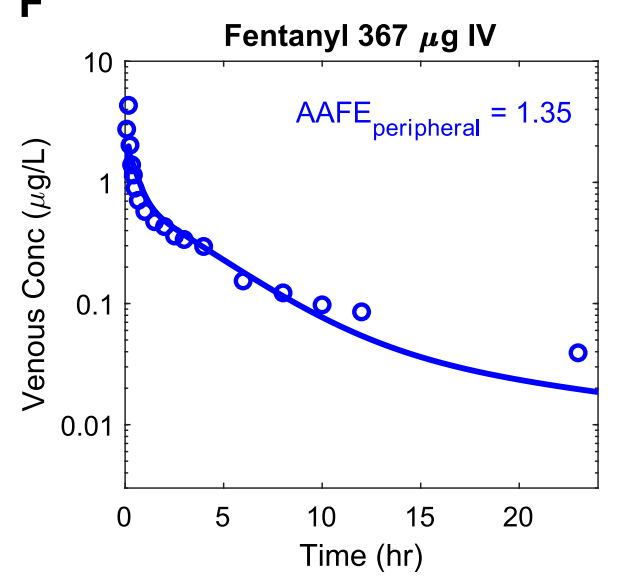

Fig. 2. Model verification using arterial and arm vein concentration profiles after intravenous (IV) administration of four test drugs. Simulated plasma drug concentration time curves and observed data are shown. (A) Nicotine sampled from the central venous compartment (right atrium), artery, and peripheral arm vein after $4.2 \mathrm{mg}$ intravenous infusion over 30 minutes. (B) Ketamine sampled from the central venous compartment, artery, and peripheral arm vein after $7 \mathrm{mg}$ intravenous infusion over 30 minutes. (C) Lidocaine sampled from the central venous compartment and artery after $210 \mathrm{mg}$ intravenous infusion over 3 minutes. (D) Lidocaine sampled from the peripheral arm vein after $105 \mathrm{mg}$ intravenous infusion over 1 hour. (E) Fentanyl sampled from the central venous compartment and artery after $25 \mu \mathrm{g}$ intravenous bolus over 5 seconds. (F) Fentanyl sampled from the peripheral arm vein after $367 \mu \mathrm{g}$ infusion over 10 minutes. Red, green, and blue curves represent simulated plasma drug concentration time curves sampled from the central venous compartment (right atrium), artery, and peripheral arm vein, respectively. Red and blue circles represent observed plasma drug concentration data sampled from the artery and arm vein, respectively. $\mathrm{AAFE}_{\text {central }}$ represents the calculated AAFE comparing the simulated central venous compartment (right atrium) drug concentration profile with the observed arterial concentration data. $\mathrm{AAFE}_{\text {peripheral }}$ represents the calculated AAFE comparing the simulated peripheral arm vein drug concentration profile with the observed arm vein concentration data. The observed data are from Tucker and Boas (1971), Gourlay and Benowitz (1997), Persson et al. (2002), Isohanni et al. (2005), Macleod et al. (2012), and Ziesenitz et al. (2015). sampling accurately represents the arterial data when lung distribution is not significant and distribution equilibrium is reached instantaneously. For nicotine and ketamine, two drugs for which observed simultaneous arterial and arm vein concentrations after intravenous administration are available, the simulated drug concentration-time profiles successfully captured the observed arteriovenous difference (Fig. 2). For these studies, the arm vein concentrations were consistently lower than the arterial concentrations before pseudo-equilibrium was reached. This difference is likely due to a forearm tissue distribution (dilution) effect, resulting in a lower $C_{\max }$ in the arm vein compared with the arterial concentration. For lidocaine and fentanyl, for which observed simultaneous arterial and arm vein concentrations were not available, separate studies that reported either arterial or arm vein concentration data were used for model verification. The independent recapitulation of either arterial or arm vein observed concentration data was successful based on the AAFE values that were within 1.5-fold (1.18-1.35). Importantly, none of these simulations of intravenous dosing applied any scalar or implemented any optimization besides top-down incorporation of CL and prediction of $\mathrm{V}_{\mathrm{ss}}$ based on observed rat $K_{\mathrm{p}}$ values. This further supports the confidence in the forearm structural model verification. As such, this work establishes the first physiologically based peripheral sampling site model that has been verified using simultaneous arterial and arm vein concentration data. The verification results suggest that our model accurately captures the arterial concentration-time profile, arm vein concentration- 
time profile, and arteriovenous difference and that the forearm structural model is sufficiently robust to be applied to simulate drug disposition for other unstudied compounds and scenarios.

Sampling Site Effect on PBPK Model-Simulated Plasma Drug Concentrations after Intravenous Administration. To investigate whether certain drug-specific PK characteristics contribute to the magnitude of the arteriovenous concentration difference after intravenous administration, the central venous compartment (right atrium), central artery, and peripheral forearm vein concentrations were simulated after intravenous administration for a matrix of hypothetical drugs (Fig. 3). PK analyses of the simulation data from each site are shown in Supplemental Table 2. The simulations unequivocally show that selection of PBPK model

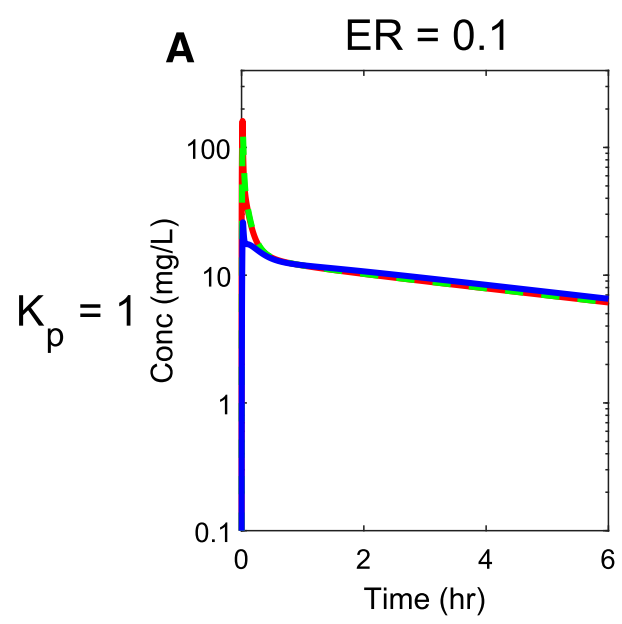

D

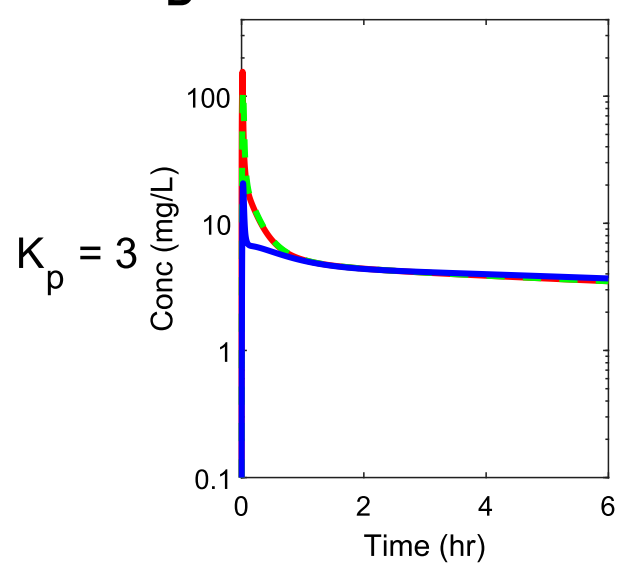

G

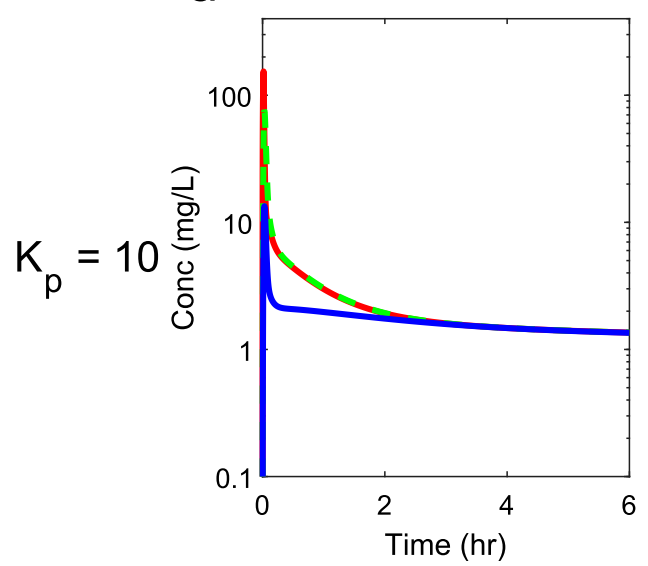

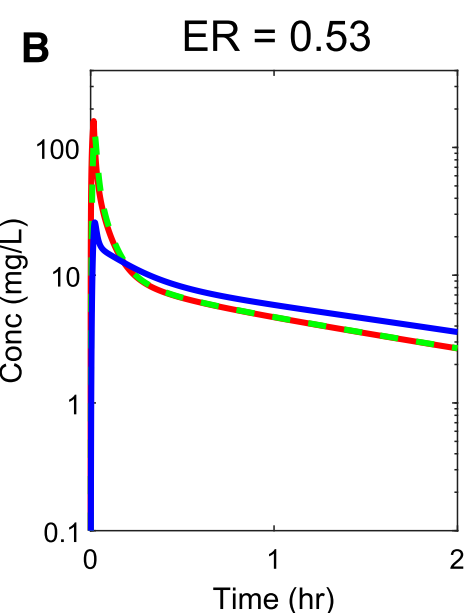

$\mathbf{E}$

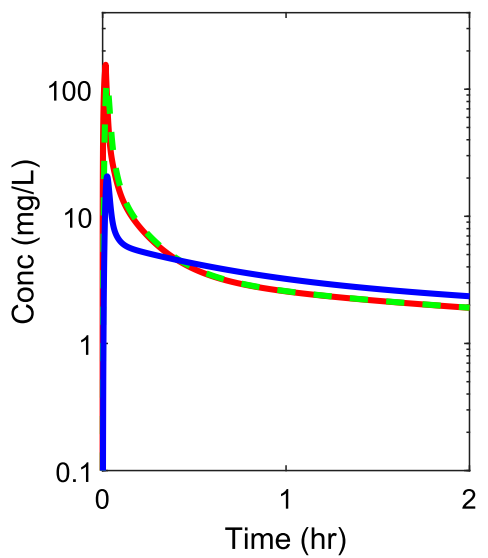

H

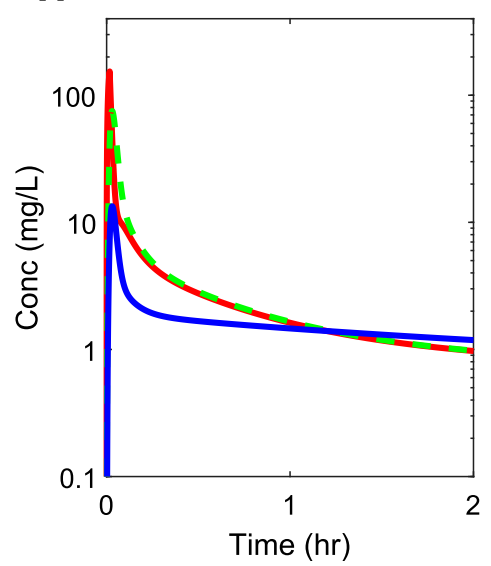

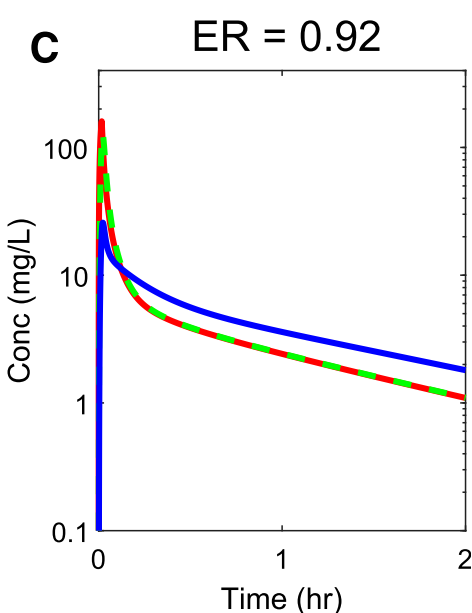

$\mathbf{F}$

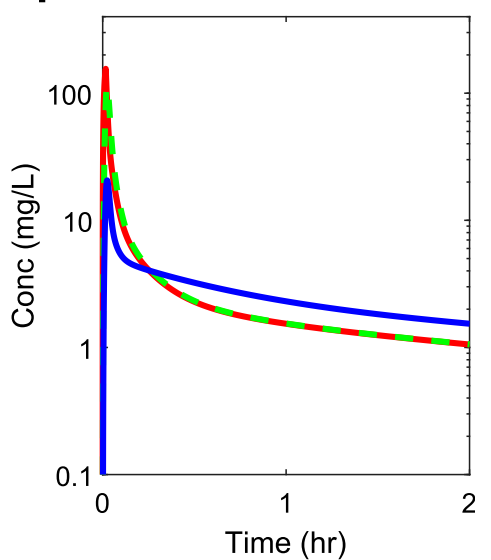

I

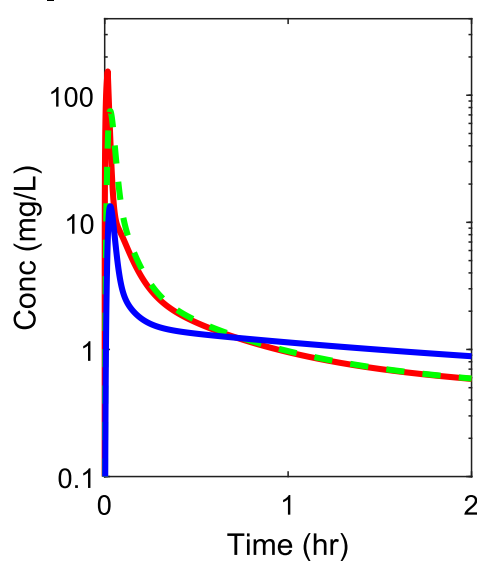

Fig. 3. Simulation of plasma drug concentration-versus-time curves after intravenous administration sampled from different sites in the PBPK model for hypothetical drugs with different clearance and distribution characteristics. (A-C) $K_{\mathrm{p}}=1$. (D-F) $K_{\mathrm{p}}=3$. (G-I) $K_{\mathrm{p}}=10$. (A, D, and G) Drugs with ER $=$ 0.1. $(\mathrm{B}, \mathrm{E}$, and H) Drugs with $\mathrm{ER}=0.53$. (C, F, and I) Drugs with $\mathrm{ER}=0.92$. All other drug parameters are as described in the Materials and Methods. In all panels, blue lines represent drug concentrations in the peripheral arm vein, red lines represent drug concentrations in the central venous compartment (right atrium), and green dashed lines represent drug concentrations in the artery. ER indicates the extraction ratio of the model drug and $K_{\mathrm{p}}$ indicates the tissue-to-plasma partition coefficient value of all tissues. All simulations were conducted as described in the Materials and Methods. 
sampling site affects the simulated distribution kinetics and the plasma concentration-time profile of the drug, and the differences between sampling sites can be considerable. However, the AUCs and CLs were equal among all sampling sites (Supplemental Table 2). As expected, the simulated central venous compartment (right atrium) concentrations and central artery concentrations were superimposable (lung distribution is not significant and distribution equilibrium in the lung is reached instantaneously). In contrast, similar to the observed studies (Fig. 2, A and B), the arm vein concentrations during the initial distribution phase and arm vein $C_{\max }$ were much lower than central venous compartment (right atrium) concentrations after intravenous dosing (Fig. 3). In addition, a slower intravenous infusion rate or longer duration of infusion did not eliminate the discrepancy in the concentrations between sampling sites (Supplemental Fig. 3). The simulations with a matrix of hypothetical drugs showed that the impact of sampling site on PK characteristics was more pronounced for drugs with high volumes of distribution (Fig. 3). Drugs with a large volume of distribution showed an appreciable concentration difference during the distribution phase where arm vein drug concentrations were consistently lower than central venous compartment (right atrium) drug concentrations. Finally, after distribution pseudo-equilibrium was reached (i.e., terminal elimination phase), the simulated peripheral arm vein concentrations were consistently higher than those collected from the central venous compartment, with drugs with higher clearances showing greater differences between the sampling sites. Taken together, these data show that special attention to the choice of experimental and simulation sampling site should be paid for drugs with large volumes of distribution or high clearances.

Sampling Site Effect on PBPK Model-Simulated Plasma Drug Concentrations after Oral Administration. Reported clinical studies investigating arteriovenous differences are almost exclusively conducted after intravenous administration. However, we hypothesized that a significant sampling site effect would also be observed after oral administration. Indeed, significant arteriovenous drug
A

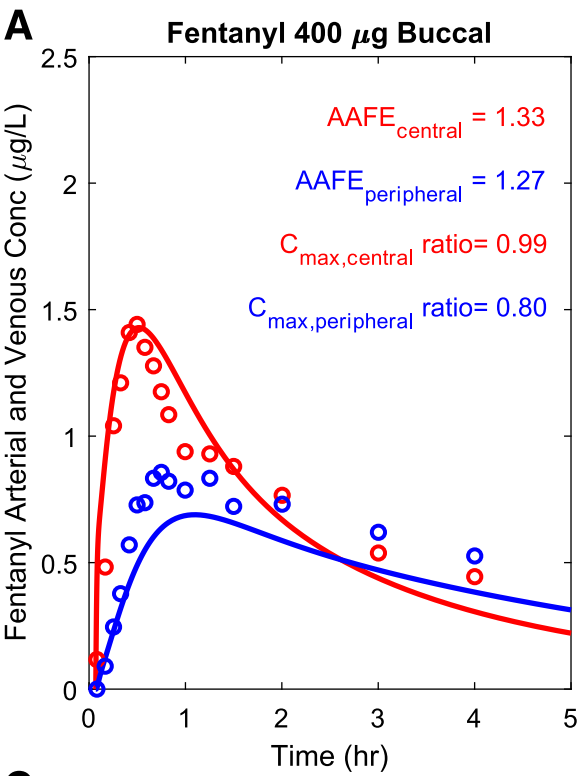

C

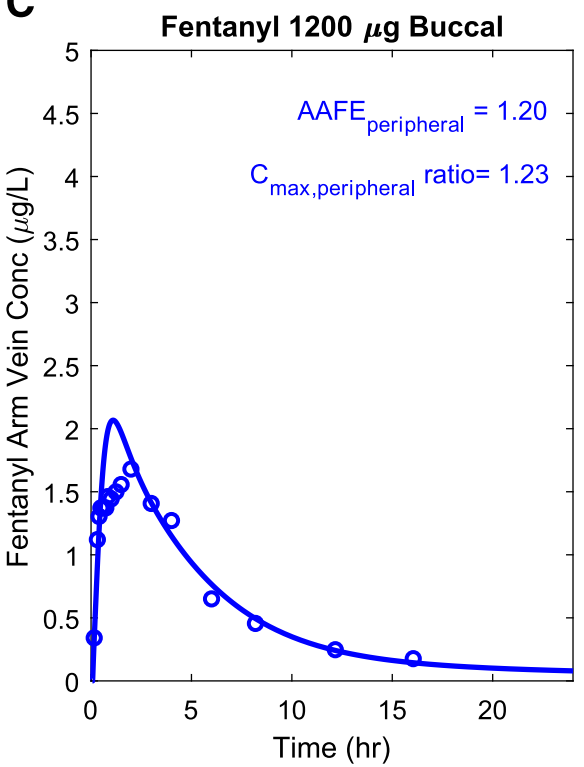

B

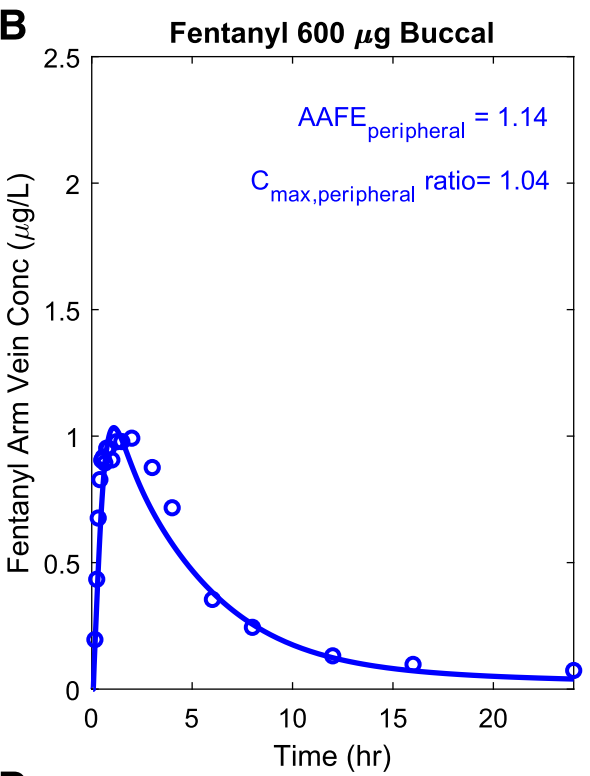

D

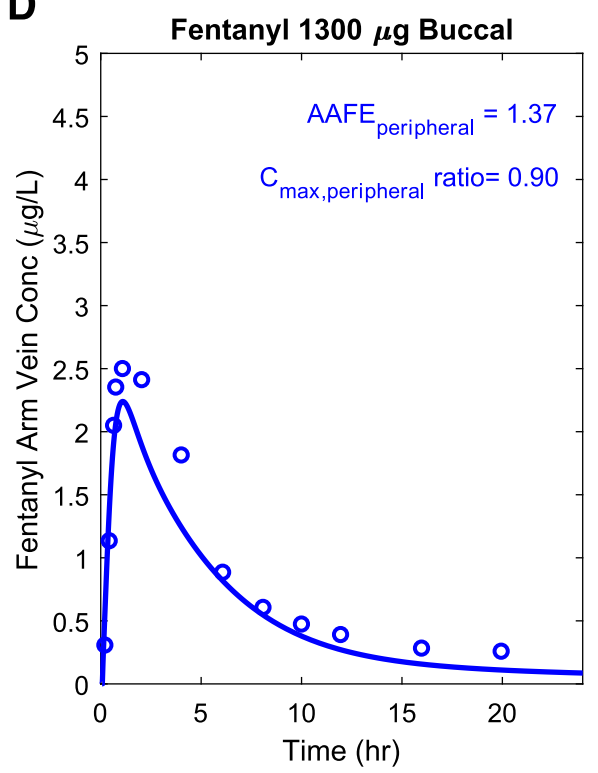

Fig. 4. Development and verification of the fentanyl buccal model using arterial and arm vein fentanyl concentration data. (A) Development of the fentanyl buccal administration model by comparing the simulated drug concentration time profiles from the central venous compartment (red curves) and peripheral arm vein (blue curves) to the observed arterial data (red circles) and observed venous data (blue circles), respectively, after $400 \mu \mathrm{g}$ buccal tablet administration. (B-D) Verification of the developed fentanyl buccal model by comparing the simulated arm vein fentanyl concentration time profiles (blue curves) to the observed arm vein fentanyl data (blue circles) after $600 \mu \mathrm{g}$ (B; Darwish et al., 2010), $1200 \mu \mathrm{g}$ (C; Darwish et al., 2006b), and $1300 \mu \mathrm{g}$ (D; Darwish et al., 2010) buccal tablet administration, respectively. $\mathrm{AAFE}_{\text {peripheral }}$ represents the calculated AAFE comparing the simulated arm vein drug concentration profile with the observed arm vein concentration data. $C_{\text {max }}$ peripheral ratio represents the ratio between the simulated maximum arm vein drug concentration and the observed maximum arm vein concentration. $\mathrm{AAFE}_{\text {central }}$ represents the calculated AAFE comparing the simulated central venous compartment (right atrium) drug concentration profile with the observed arterial concentration data. $C_{\max }$, central ratio represents the ratio between the simulated maximum central venous compartment drug concentration and the observed maximum arterial concentration. 
concentration differences were shown for fentanyl after buccal administration (Darwish et al., 2006a). To explore this difference, the fentanyl model verified after intravenous dosing (Fig. 2) was further developed and verified for buccal administration (Fig. 4). To simulate buccal administration, the buccal absorption rate constant $\left(k_{\text {a,buccal }}\right)$ and the gut absorption rate constant $\left(k_{\mathrm{a} \text {,gut }}\right)$ were incorporated into the model and optimized to be 1 and 3 hours $^{-1}$, respectively, based on the observed fentanyl arteriovenous difference (Fig. 4A). To verify the optimized buccal absorption rate constant $\left(k_{\text {a,buccal }}\right)$ and gut absorption rate constant $\left(k_{\mathrm{a}, \text { gut }}\right)$, three independent studies were used as test data sets (Fig. 4, B-D). As shown in Fig. 4, the optimized absorption rate parameters successfully recapitulated all independent test data sets sampled from the arm vein with all AAFE values $<2$ and all simulated $C_{\max }$ values within the 0.8 - to 1.25 -fold range of observed $C_{\max }$ values (Fig. $4, \mathrm{~B}-\mathrm{D}$ ). Hence, the optimized absorption rate constants were considered successfully verified. Such verification substantiates the validity of our forearm structural model and the applicability of our model to simulate arteriovenous differences after oral administration. The data with fentanyl after buccal administration (Darwish et al., 2006a) also strongly suggest that the importance of arteriovenous differences is not limited to intravenous administration.

To investigate the overall effect of sampling site on arteriovenous concentration difference after oral administration, the verified model was used to simulate arterial and arm vein drug concentrations after oral dosing for a matrix of drugs with a variety of PK parameters (Fig. 5). Similar to the intravenous dosing, the simulations of oral administration show that selection of the PBPK model sampling site affects the simulated drug distribution kinetics and the plasma drug concentrationtime profile, but not AUCs (Supplemental Table 3). Specifically, the arm vein concentrations during the initial distribution phase were lower than the central venous compartment (right atrium) concentrations, and slower oral absorption rates did not eliminate this discrepancy (Supplemental Fig. 4). This impact of sampling site during the initial distribution phase was more pronounced for drugs with large volumes of distribution. The $K_{\mathrm{p}}$ values of skin, adipose, and muscle, which directly affect the forearm distribution kinetics, had a sizable effect on the concentration difference between the arm vein and the central venous compartment (right atrium) (Fig. 5, J-O). As a result, the values for $C_{\max }$ and time taken to reach $C_{\max }\left(t_{\max }\right)$ were substantially affected. The simulated $C_{\max }$ value obtained by sampling from the central venous compartment was higher than the $C_{\text {max }}$ obtained from the peripheral arm vein, with a $C_{\max }$ ratio between the two sites ranging from 1.07 to 4.25 (Fig. 5). The simulated $t_{\max }$ value for the drug based on central venous sampling was consistently shorter (0.12- to 0.72 -fold) than the $t_{\max }$ from peripheral arm vein sampling (Fig. 5). Similar to the intravenous dosing, during the terminal elimination phase, the simulated arm vein concentrations of the drug were higher than those collected from the central venous compartment, and drugs with higher clearances showed greater differences (Supplemental Table 3). Taken together, these simulations show that the choice of sampling site also has a quantitatively important effect after oral administration on clinically significant measures of drug exposure and accumulation such as $C_{\max }$ and $C_{\text {trough }}$.

Sampling Site Effect on PBPK Model Evaluation, Parameter Optimization, and Model Extrapolation.
During PBPK model development, simulated central venous compartment (right atrium) concentrations are usually compared with observed PK data that are almost exclusively obtained from the peripheral arm vein, and the model parameters are optimized to achieve concordance between simulated and observed data. Because of the true differences between central venous compartment (right atrium) and peripheral arm vein concentrations, we hypothesized that the use of the simulated central venous compartment concentration-time profile and observed arm vein concentrations may result in biased model evaluation despite the use of verified "true" parameters. This hypothesis was tested using the verified fentanyl buccal and intravenous administration model (Fig. 6). Although the verified absorption rate parameters correctly simulated peripheral and arterial fentanyl concentrations (Fig. 4), the use of the central venous compartment (right atrium) sampling site as the PBPK model output with observed arm vein data rendered all simulations unacceptable with $\mathrm{AAFE}$ values $>2$ and $C_{\max }$ ratios outside the 0.8- to 1.25-fold range (Fig. 6). As a result, when the discrepant sampling site is used, the true drug parameters are likely not selected for the PBPK model under development (Fig. 7A). Instead, false model parameters will be obtained through erroneous model optimization until the simulated central venous profile conforms to the observed arm vein data (Fig. 7B). The difference between the "true" and "false" parameter estimates can be considerable, as illustrated by the fentanyl example. Whereas the verified "true" parameters for $k_{\text {a,buccal }}$ and $k_{\mathrm{a} \text {,gut }}$ were 1 and 3 hours $^{-1}$, respectively (Fig. 4), the erroneously optimized $k_{\mathrm{a} \text {,buccal }}$ and $k_{\text {a,gut }}$ for central venous sampling were 0.35 and 0.7 hours $^{-1}$ respectively (Fig. $7 \mathrm{~B}$ ). This illustrates that parameter optimization using the simulated central venous profile and observed arm vein sampling may result in confounded drug models that do not accurately reflect the true PK properties.

To test whether the erroneous parameter optimization has an impact in model application and whether the use of discrepant sampling sites between simulation and observation can result in biased prediction in unstudied clinical scenarios, a hypothetical case with increased $k_{\text {a,buccal }}$ (new formulation) for fentanyl was simulated using the model optimized based on arm vein ("true") (Fig. 7C) and central venous sampling ("false") (Fig. 7D). The $C_{\text {max }}$ in the arm vein due to the new formulation was predicted to increase by $40 \%$ (Fig. 7C) using the verified arm model and verified absorption parameters, whereas the $C_{\max }$ using central venous sampling and erroneous parameters was predicted to increase by $119 \%$ (Fig. 7D). This shows an approximately 3 -fold difference in the predicted magnitude of formulation effect on $C_{\max }$, which would be considered clinically significant. The simulations show that PBPK models developed and optimized using central venous sampling can erroneously predict the impact of new formulation on PK and drug disposition and can lead to incorrect conclusions that have clinical significance.

Sampling Site Effect on Simulated Plasma Metabolite Concentration Using a PBPK Model. An aspect that has not been clinically tested in humans is whether sampling site has an impact on metabolite plasma concentration profile. To investigate the overall sampling site effect on plasma metabolite concentration, the central venous compartment (right atrium) and peripheral forearm vein were sampled for simulated metabolite concentrations after intravenous (Fig. 8) and oral (Fig. 9) administration of the parent drug. The simulation data show that selection of the sampling site 
affects the simulated distribution kinetics and the plasma concentration-time profile of the metabolite as well, but not the metabolite AUC. Similar to the parent drug, the impact of sampling site on metabolite simulation was more pronounced with metabolites with high volumes of distribution (high $K_{\mathrm{p}, \mathrm{m}}$, Figs. 8 and 9 ). The simulated metabolite $C_{\max }$ value obtained by sampling from the central venous compartment was in general higher than the metabolite $C_{\max }$ obtained from the arm vein, with a $C_{\max }$ ratio between the two sites ranging from 1.00 to 4.62 (Figs. 8 and 9).

Intriguingly, the choice of the sampling site in PBPK simulations of metabolites had some unexpected effects. A metabolite
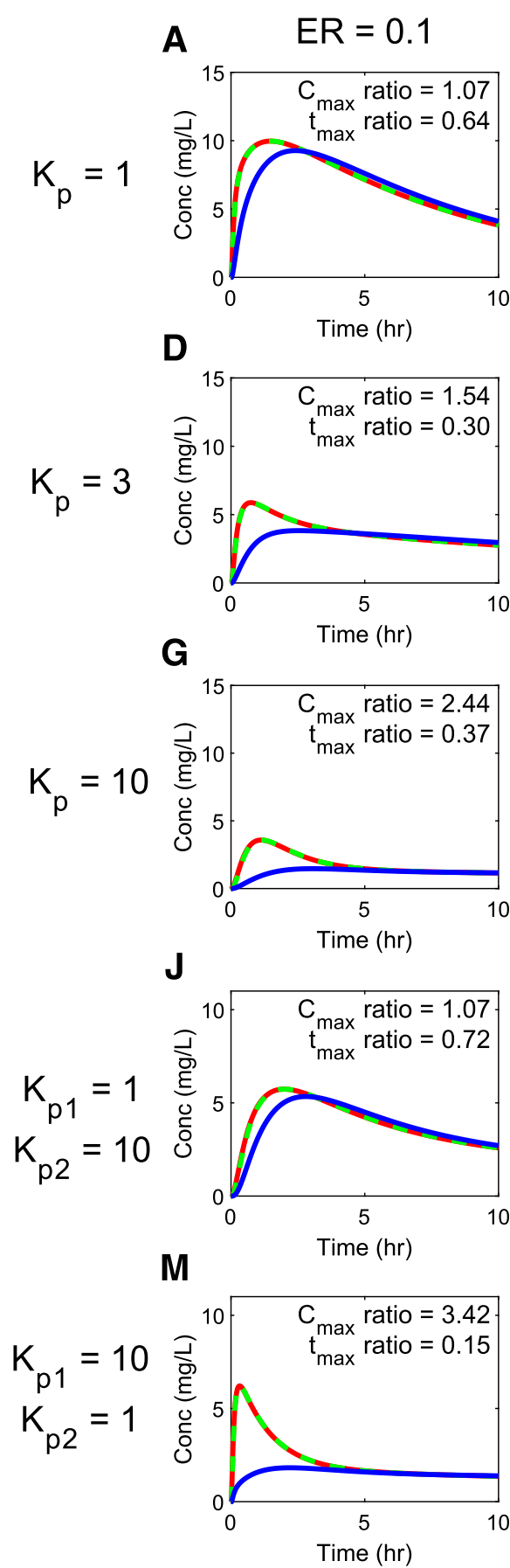

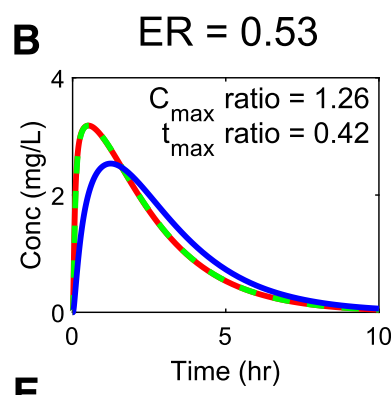

E

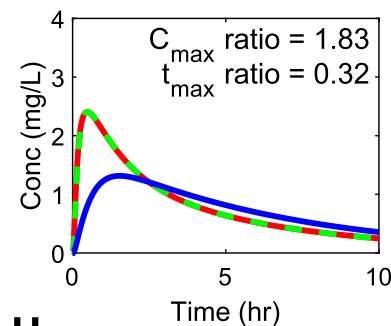

H
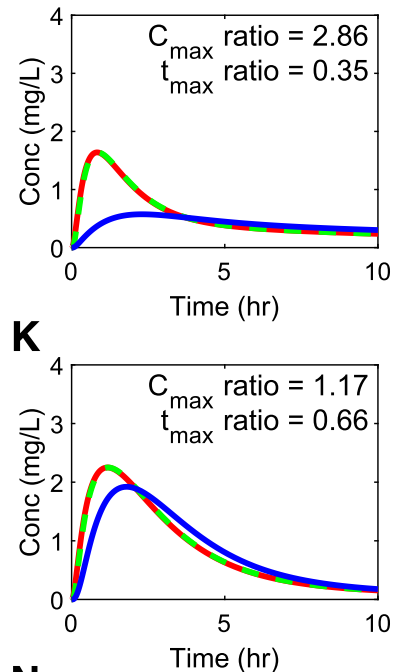

$\mathbf{N}$

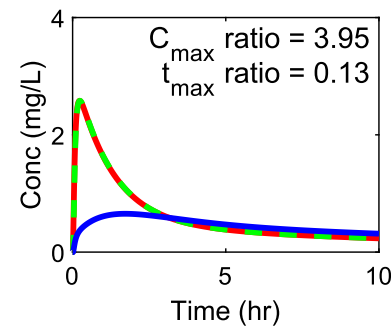

C $\quad \mathrm{ER}=0.92$
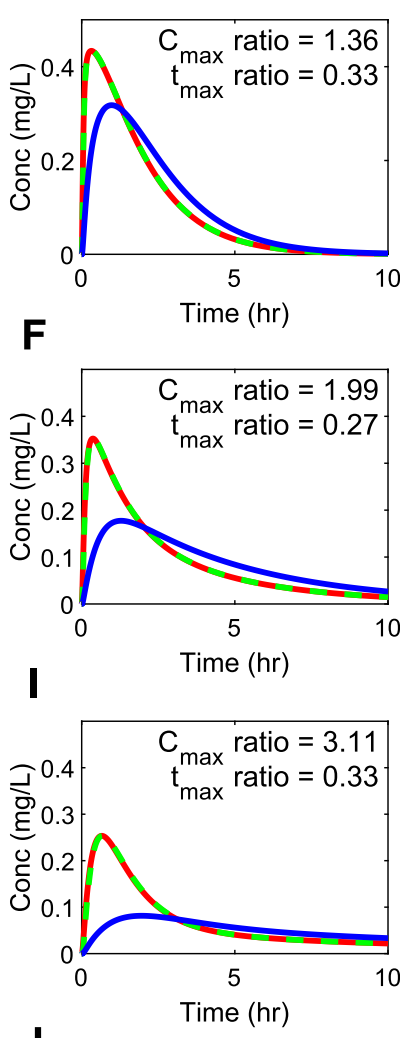

$\mathbf{L}$
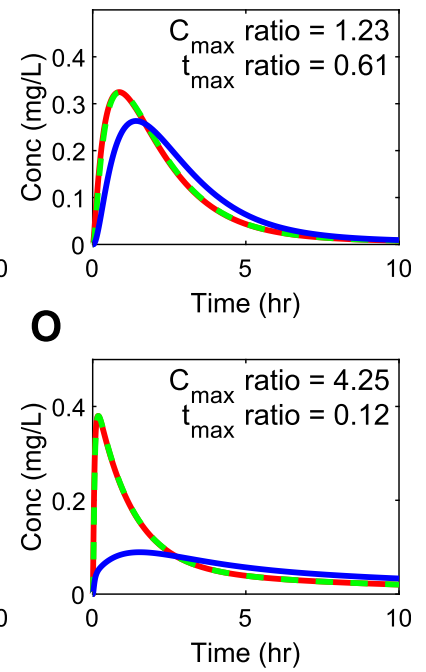

Fig. 5. Simulation of plasma drug concentration-versus-time curves after oral administration sampled from different sites in the PBPK model for hypothetical drugs with different clearance and distribution characteristics. (A-C) $K_{\mathrm{p}}=1$. (D-F) $K_{\mathrm{p}}=3$. (G-I) $K_{\mathrm{p}}=10$. (J-L) $K_{\mathrm{p}}$ value of adipose, skin,

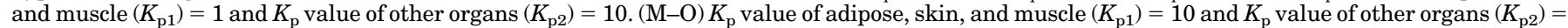

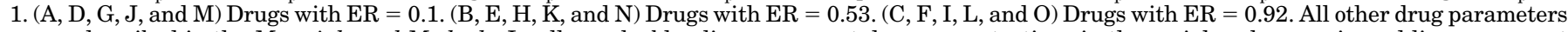

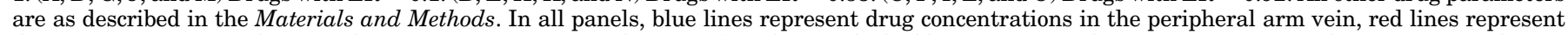

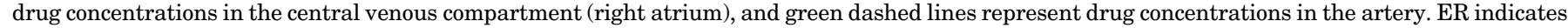

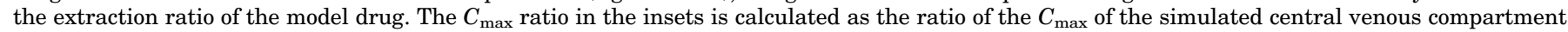

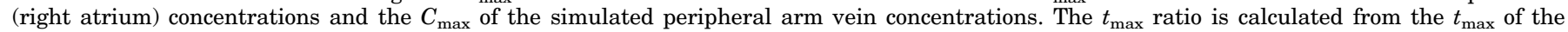
simulated central venous compartment concentrations and the $t_{\max }$ of the simulated arm vein concentrations. 


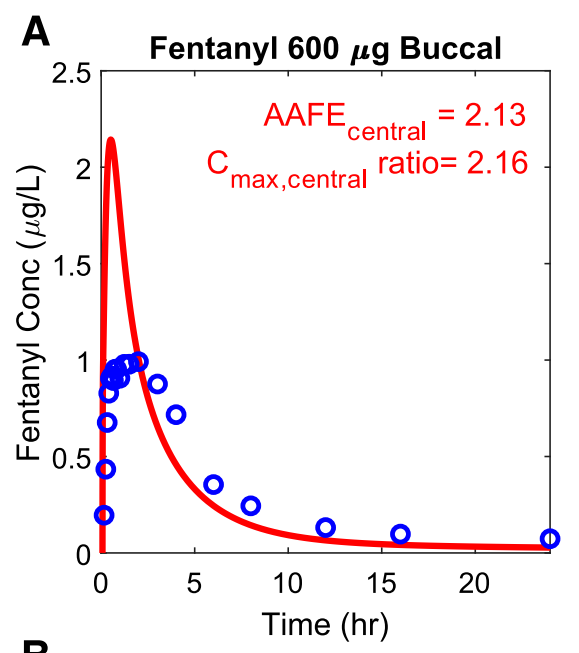

B

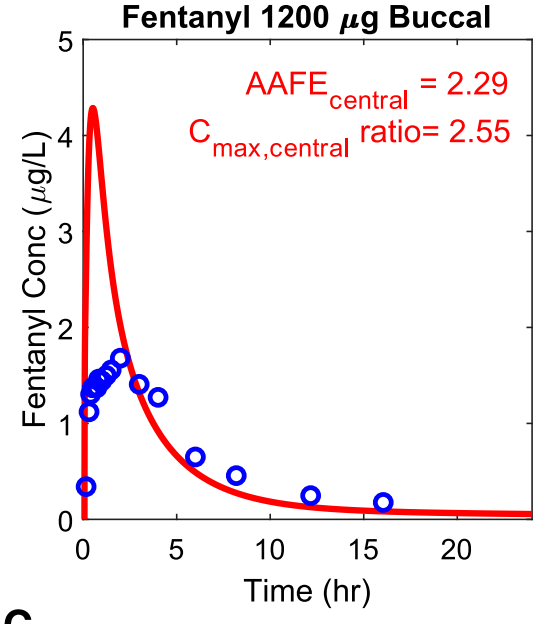

C

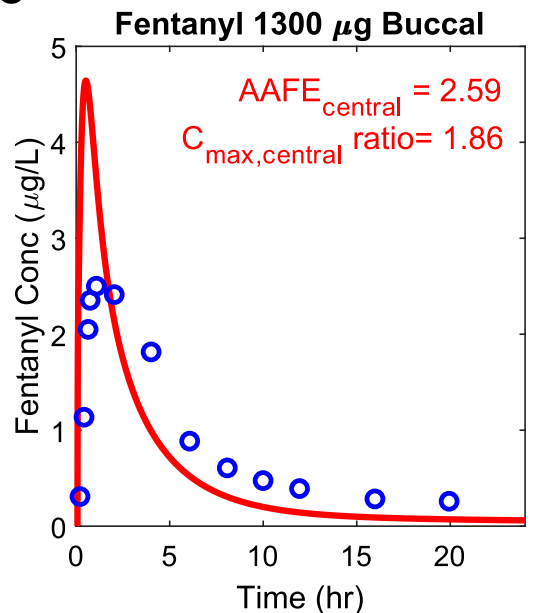

Fig. 6. Impact of the choice of sampling site on PBPK model evaluation. (A-C) The impact of discrepant sampling site between observed and simulated data on model performance evaluation. The simulated drug concentration time profiles (red curves) are conventional PBPK simulation outputs sampled from the central venous compartment (right atrium) and the observed data are from commonly used arm vein sampling (blue circles) after $600 \mu \mathrm{g}$ (A; Darwish et al., 2010), $1200 \mu \mathrm{g}$ (B; Darwish et al., 2006b), and $1300 \mu \mathrm{g}$ (C; Darwish et al., 2010) buccal tablet administration of fentanyl, respectively. The observed data shown and the model used here are the same as those shown and used in Fig. 4. $\mathrm{AAFE}_{\text {central }}$ represents the calculated AAFE comparing the simulated central venous compartment (right atrium) drug concentration profile with the observed arm vein concentration data. $C_{\max \text {, central }}$ ratio represents the ratio between the simulated maximum central venous compartment (right atrium) drug concentration and the observed maximum arm vein concentration. plasma concentration-time profile is expected to follow a biphasic curve of increasing and decreasing plasma concentrations. However, metabolite concentrations sampled from the central venous compartment (right atrium) showed a unique profile with a spike in the metabolite concentrations during the distribution phase of the parent drug after intravenous dosing (Fig. 8, A, D, and G) followed by a second peak of the plasma concentrations corresponding to the metabolite $C_{\max }$ expected from arm vein sampling. The presence of the spike could also be observed after oral dosing when the drug had a low clearance (Fig. 9, D and G) but was less pronounced compared with intravenous dosing. Furthermore, the spike observed from the central venous (right atrium) profile was hardly observed from the arm vein profile, which agrees with the observed metabolite profile from common clinical studies.

\section{Discussion}

It has been shown that arterial drug concentrations can be remarkably different from peripheral venous drug concentrations in animals and humans (Chiou, 1989; Gourlay and Benowitz, 1997; Ericsson et al., 2000; Rentsch et al., 2001; Persson et al., 2002; Darwish et al., 2006a; Olofsen et al., 2010). The physiologically based simulations presented here reproduced the observed phenomenon of arteriovenous drug concentration differences. To our knowledge, our peripheral sampling model is the first physiologically based model that is verified using both arterial and arm vein drug concentrations simultaneously. Our analysis is also the first to systematically evaluate drug PK characteristics that drive arteriovenous drug concentration differences after both intravenous and oral administration. Consistent with previous theory (Chiou, 1989), the simulations show that arteriovenous differences during the initial distribution phase are most pronounced for compounds with large volumes of distribution, since the arteriovenous difference during the initial phase is due to drug distribution into the forearm tissue. During the terminal elimination phase, the arteriovenous differences are only significant for compounds with high clearances, as the arteriovenous difference during the terminal phase is a result of the blood emerging from the eliminating organ diluting the drug concentration measured in the central venous compartment (right atrium). The simulations also show that arteriovenous differences in drug concentrations can be significant after oral administration. This is important because minimal clinical studies have been done to explore arteriovenous differences after oral administration in humans. Yet PBPK modeling is frequently used to simulate oral dosing scenarios and to specifically explore the $C_{\max }$. Based on our simulations and the existing data (Gourlay and Benowitz, 1997; Moksnes et al., 2008), it is highly likely that arteriovenous differences after other routes of administration (e.g., intranasal or inhalation) are also important and should be considered in PBPK modeling of these routes of administration. In addition, our PBPK modeling approach could be extended to preclinical species in which experimental studies with various sampling sites and routes of administration are feasible and the model predictions can be verified.

Based on the observed arteriovenous differences, we hypothesized that this difference would be crucial for PBPK modeling when the model does not output simulations from the same site as the observed study. One of the key advantages of PBPK models 
A
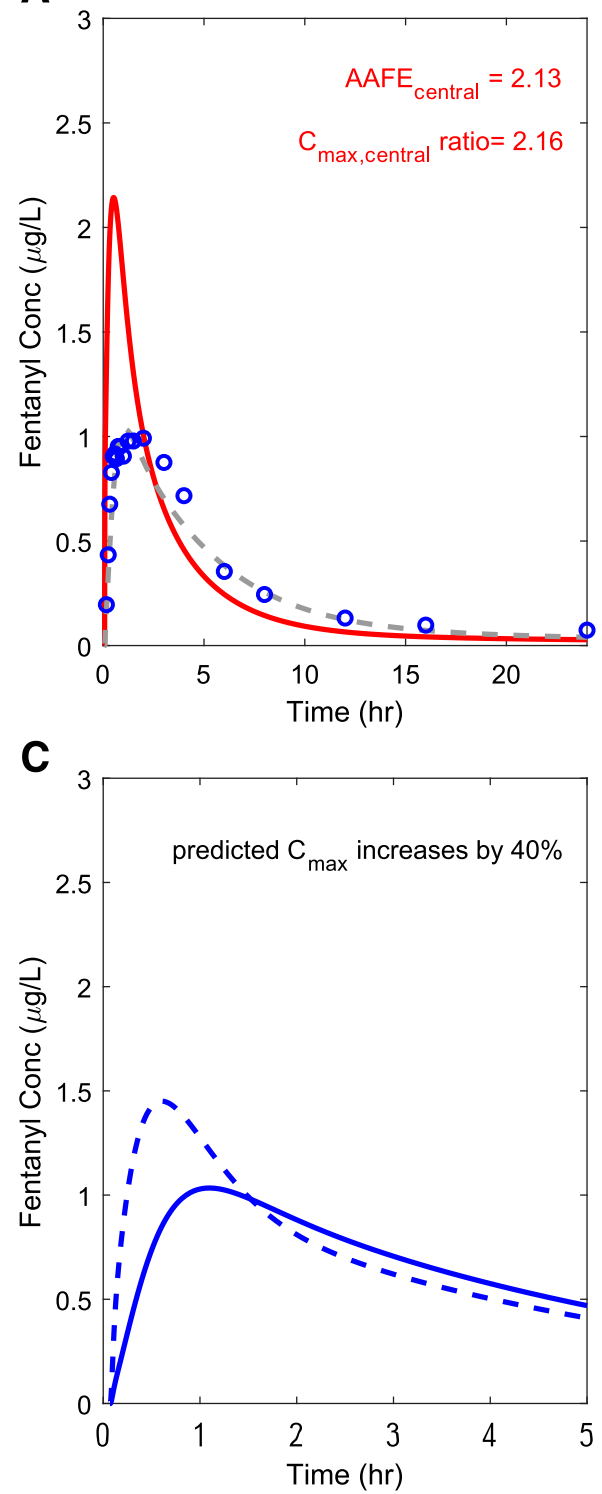

B

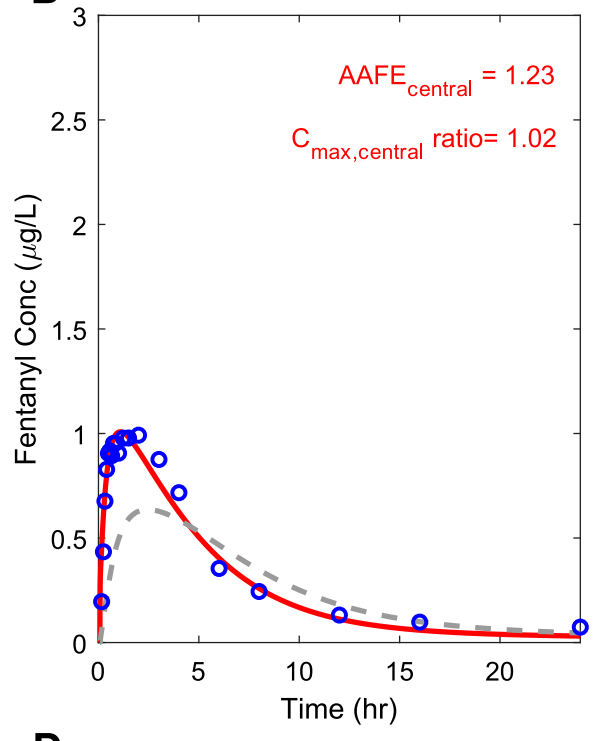

D

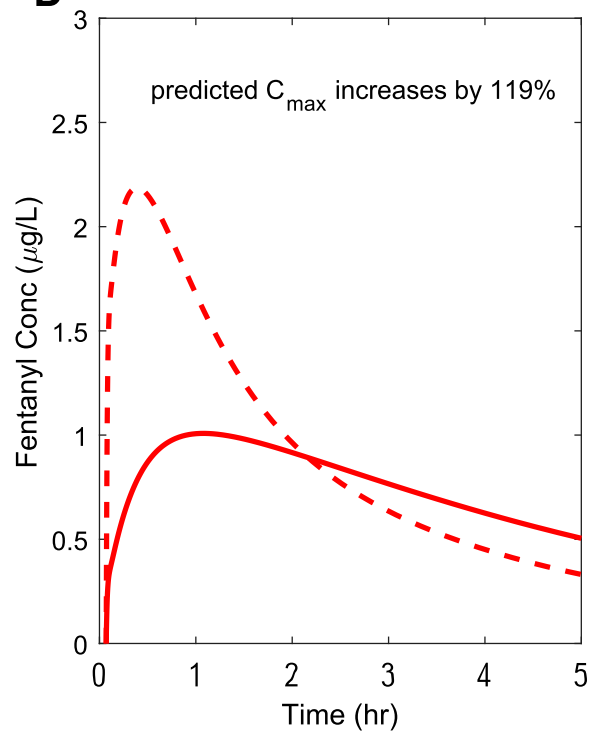

Fig. 7. Impact of the choice of sampling site on PBPK model parameter optimization and extrapolation to unstudied clinical scenarios. (A) A scenario in which the modeler uses the PBPK model-simulated central venous compartment (right atrium) concentrations (red curve) for comparison with the observed data from the peripheral arm vein (blue circles), rather than using the simulated peripheral sampling site concentrations (gray curve shown for comparison). In this case, both $\mathrm{AAFE}$ and $C_{\max }$ ratio suggest model rejection although true parameters were used $\left(k_{\mathrm{a}, \mathrm{buccal}}\right.$ $=1$ hour $^{-1}$ and $k_{\mathrm{a} \text {,gut }}=3$ hours $^{-1}$ ). (B) Fentanyl simulation with $k_{\text {a,buccal }}=0.35$ hours $^{-1}$ and $k_{\mathrm{a} \text {,gut }}=0.7$ hours $^{-1}$ based on erroneous parameter optimization to adapt simulated central venous concentrations to conform to observed data collected from arm vein. The gray line shows the simulated arm vein concentrations using the same "false" parameters. (C) Simulation of the impact of a new formulation with a $400 \%$ increase in $k_{\mathrm{a}, \text { buccal }}$ (dashed blue curve) on the plasmaconcentration curve sampled from the arm vein in comparison with baseline $k_{\mathrm{a} \text {,buccal }}$ (solid blue curve) using the verified peripheral sampling site model with true parameters, predicting a $40 \%$ increase in arm vein $C_{\text {max }}$. (D) Simulation of the same new formulation as in (C) with a $400 \%$ increase in $k_{\text {a buccal }}$ (dashed red curve) in comparison with baseline $k_{\text {a,buccal }}$ (solid red curve) using central venous compartment sampling with the erroneously optimized parameters from (B). The central venous compartment sampling predicts a $119 \%$ increase in $C_{\text {max }}$. is that they allow simulation of complete plasma concentrationtime curves and especially $C_{\max }$ for drugs with narrow therapeutic indexes that have concerns of concentration-dependent adverse effects. Therefore, it is important to address whether correct simulated concentrations (appropriate compartment) are sampled from PBPK models to reflect the concentrations driving pharmacological or toxicological effects. Our findings highlight the need for either sampling from the arm artery in experimental PK studies and comparing the data to PBPK model-simulated arterial concentrations, or sampling from the arm vein in studies and comparing the data to simulated peripheral sampling compartment concentrations. For the latter, our results suggest that a peripheral sampling compartment that specifically addresses distribution at a peripheral sampling site needs to be developed and incorporated into PBPK models to allow meaningful comparisons between simulated and observed drug concentrations.

In this study, an in silico peripheral sampling compartment was developed using human forearm physiology to replicate the peripheral sampling site. In contrast, previous models
(Levitt, 2004; Musther et al., 2015) used an optimized hybrid of emergent veins from total body skin, adipose, and muscle tissue without constructing a physiologically based peripheral sampling compartment. Compared to the previous hybrid models, the physiologically based model developed here performed better, showing successful simulations of all verification data sets (AAFE 1.16-1.35) without extensive drug parameter optimization. The first study (Levitt, 2004) used an optimized hybrid approach and could not generate a standardized arm model. Different arm model parameters were needed to recapitulate disposition for different chemicals. The second study (Musther et al., 2015) modified Levitt's model and used the simulated/observed $C_{\max }$ ratio as the evaluation criteria. Only 6 of 15 test data sets had a simulated/observed $C_{\max }$ ratio within 0.8 - to 1.25 -fold and the model was not validated using arterial and arm vein concentration data simultaneously. One uncertain parameter in all of these models is the anastomoses fraction. Due to incomplete physiological information of anastomoses in humans, a $10 \%$ fraction was estimated previously and used here. This is likely an acceptable parameter, as the 


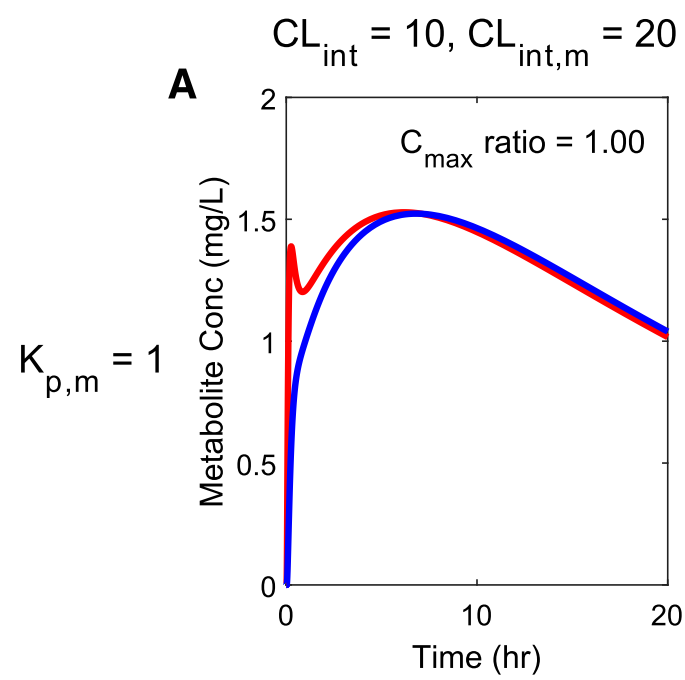

D
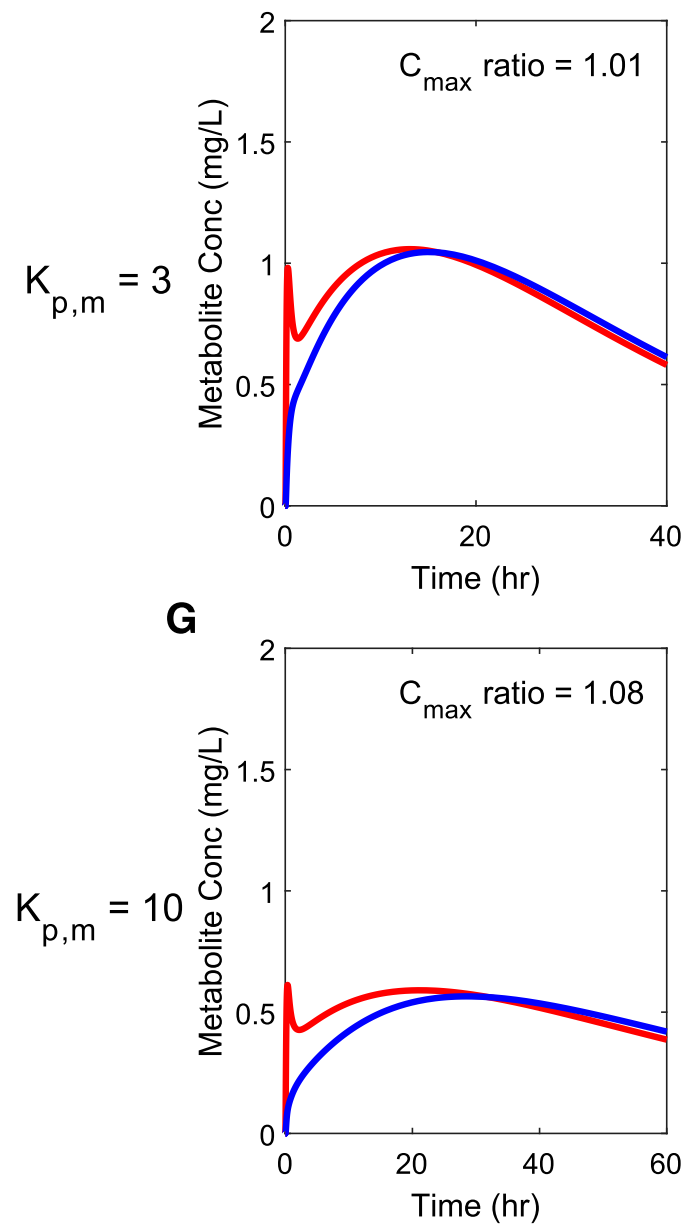

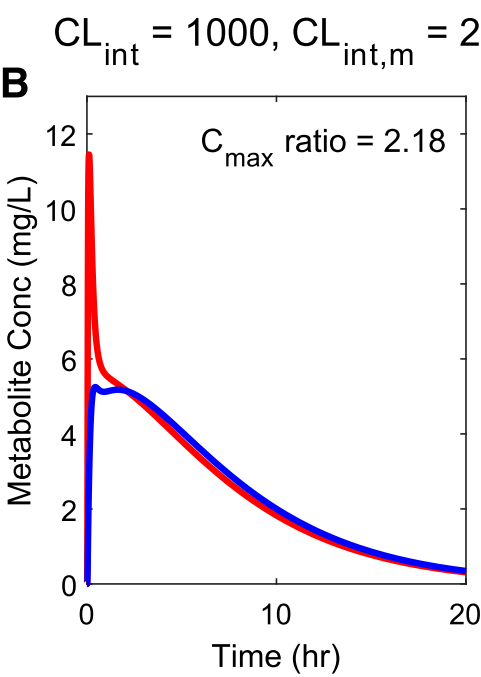

$\mathbf{E}$

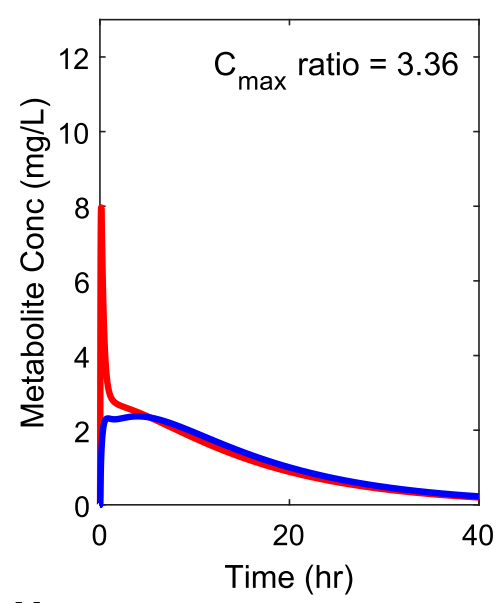

H

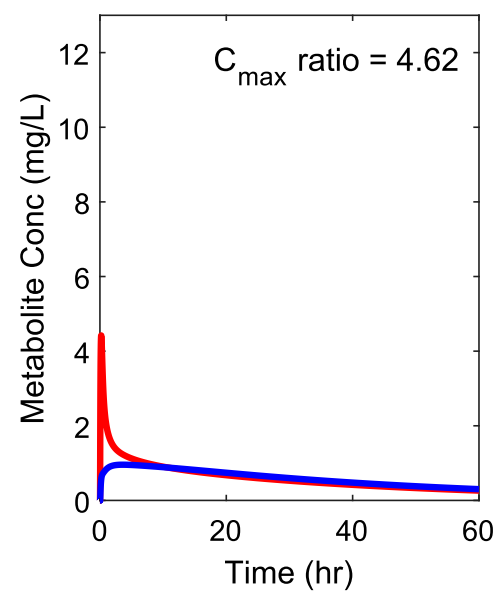

$\mathrm{CL}_{\text {int }}=10, \mathrm{CL}_{\text {int, } \mathrm{m}}=2000$

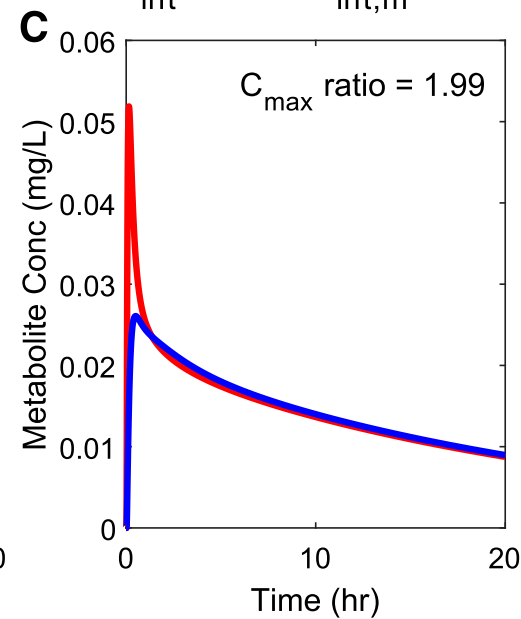

$\mathbf{F}$

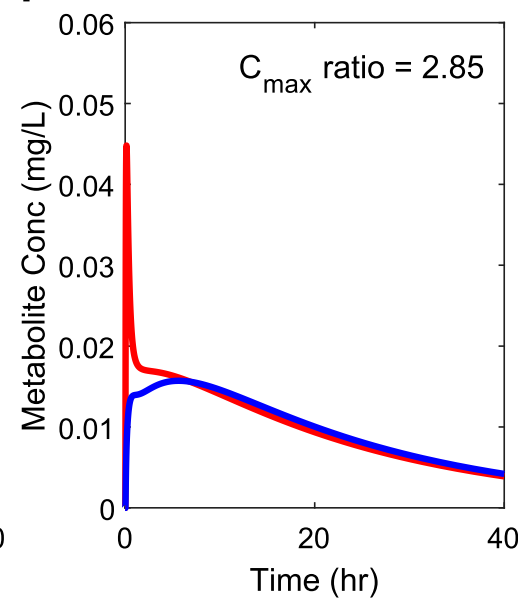

I

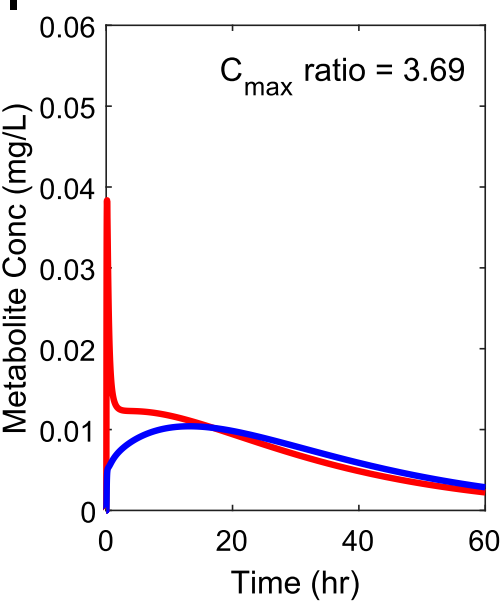

Fig. 8. Simulated plasma metabolite concentration-versus-time curves after intravenous administration of parent drug sampled from different sites in the PBPK model for metabolites with different clearance and distribution characteristics. (A-C) Metabolite $K_{\mathrm{p}}\left(K_{\mathrm{p}, \mathrm{m}}\right)=1$. $(\mathrm{D}-\mathrm{F}) K_{\mathrm{p}, \mathrm{m}}=3$. $(\mathrm{G}-\mathrm{I}) K_{\mathrm{p}, \mathrm{m}}=$ 10. (A, D, and G) Metabolite concentration curves when drug $\mathrm{CL}_{\text {int }}=10 \mathrm{l} /$ hour and $\mathrm{CL}_{\mathrm{int}, \mathrm{m}}=20 \mathrm{l} / \mathrm{hour}$. (B, E, and $\mathrm{H}$ ) Metabolite concentration curves when drug $\mathrm{CL}_{\text {int }}=1000$ l/hour and $\mathrm{CL}_{\text {int, }}=20$ l/hour. $\left(\mathrm{C}, \mathrm{F}\right.$, and I) Metabolite concentration curves when drug $\mathrm{CL}_{\text {int }}=10 \mathrm{l} / \mathrm{hour}$ and $\mathrm{CL}_{\text {int,m }}=2000 \mathrm{l} /$ hour. The drug $K_{\mathrm{p}}$ value of all organs equals 3 in all panels. In all panels, blue lines represent metabolite concentrations in the peripheral arm vein, and red lines represent metabolite concentrations in the central venous compartment (right atrium). The $C_{\max }$ ratio in each panel is calculated using the $C_{\text {max }}$ of the simulated central venous compartment (right atrium) metabolite concentration divided by the $C_{\max }$ of the simulated arm vein metabolite concentration.

sensitivity analysis (Supplemental Fig. 1) showed that it is not a critically sensitive parameter. Commercial PBPK software such as PK-Sim or Simcyp also provide the option to sample from a peripheral site, with Simcyp using the published model
(Musther et al., 2015). However, whether commercial software users employ this during modeling is unclear. Although our forearm sampling model is distinctly different from the Simcyp model, the discrepancy between simulated central and peripheral 

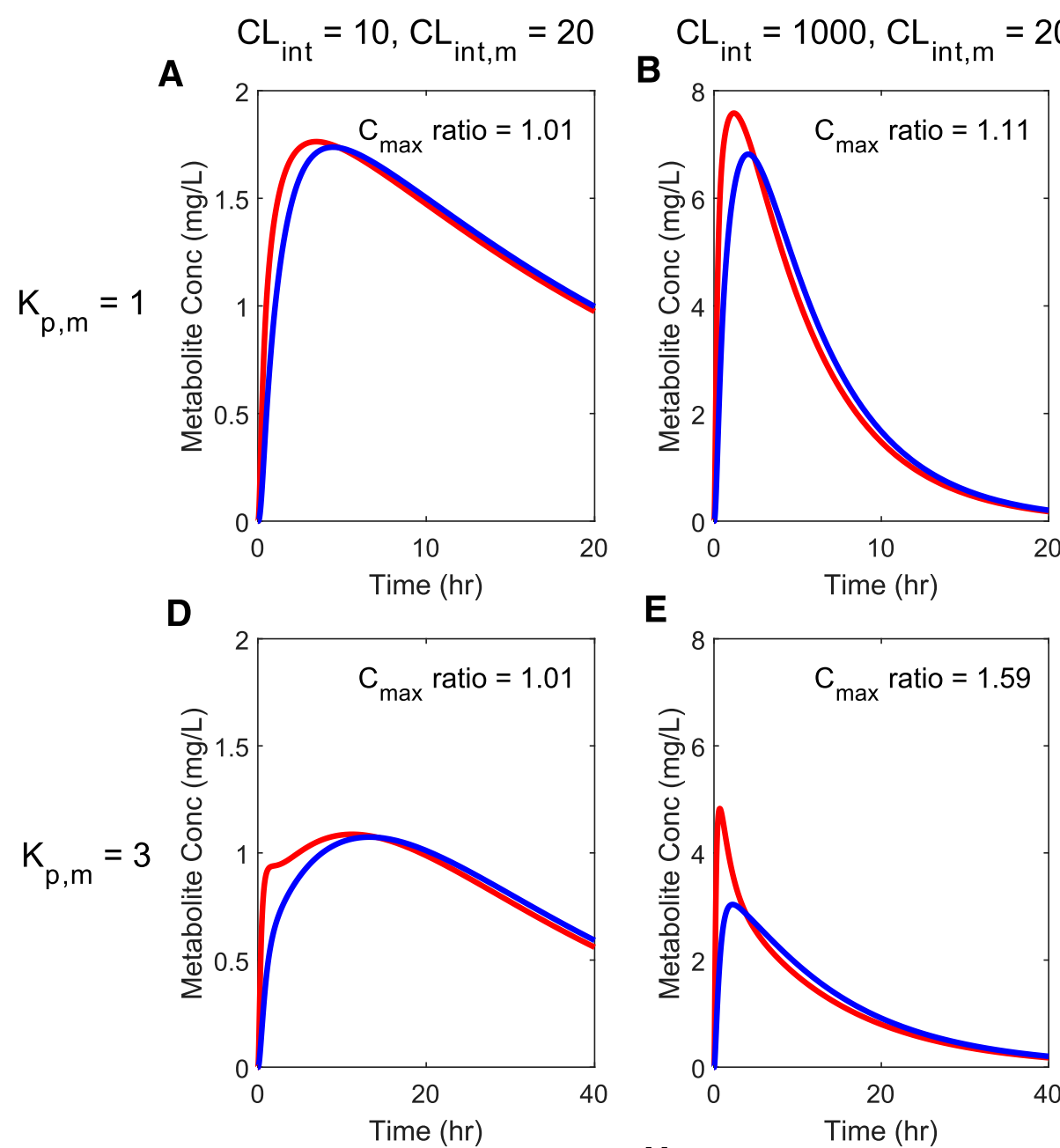

E
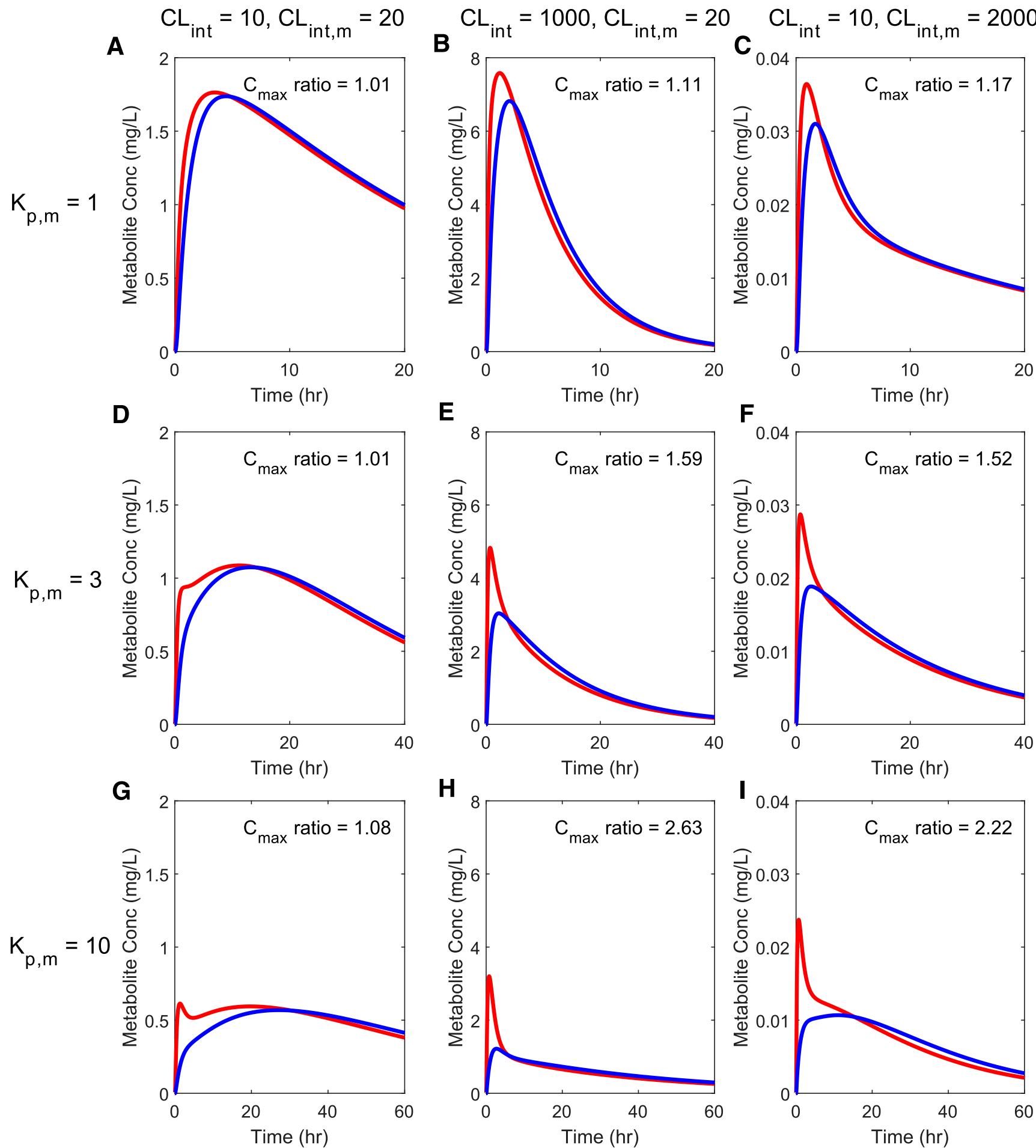

Fig. 9. Simulated plasma metabolite concentration-versus-time curves after oral administration of drug with PBPK models for metabolites with different clearance and distribution characteristics. (A-C) Metabolite $K_{\mathrm{p}}\left(K_{\mathrm{p}, \mathrm{m}}\right)=1$. (D-F) $K_{\mathrm{p}, \mathrm{m}}=3$. (G-I) $K_{\mathrm{p}, \mathrm{m}}=10$. (A, D, and G) Metabolite concentration curves when drug $\mathrm{CL}_{\text {int }}=10 \mathrm{l} /$ hour and $\mathrm{CL}_{\text {int,m }}=20 \mathrm{l} / \mathrm{hour}$. (B, E, and $\mathrm{H}$ ) Metabolite concentration curves when drug $\mathrm{CL}_{\text {int }}=1000 \mathrm{l} / \mathrm{hour}$ and $\mathrm{CL}_{\text {int,m }}=20 \mathrm{l} / \mathrm{hour}$. (C, F, and I) Metabolite concentration curves when drug $\mathrm{CL}_{\text {int }}=10 \mathrm{l} / \mathrm{hour}$ and $\mathrm{CL}_{\mathrm{int}, \mathrm{m}}=2000 \mathrm{l} / \mathrm{hour}$. The drug $K_{\mathrm{p}}$ value of all organs equals 3 in all panels. In all panels, blue lines represent metabolite concentrations in the arm vein, and red lines represent metabolite concentrations in the central venous compartment (right atrium). The $C_{\max }$ ratio in each panel is calculated using the $C_{\max }$ of the simulated central venous compartment (right atrium) metabolite concentration divided by the $C_{\max }$ of the simulated arm vein metabolite concentration.

concentrations for both drug and metabolite after intravenous and oral dosing was qualitatively replicated using Simcyp version 17 (Supplemental Fig. 5). Therefore, this phenomenon appears to be a general consideration for all PBPK models. More attention should be paid to specifying and reporting the sampling site in PBPK modeling regardless of the modeling platform used. For establishing compound-specific drug models, it is advisable to experimentally determine the $K_{\mathrm{p}}$ values for 
muscle (most sensitive), skin, and adipose to capture sampling site distribution. If this is not possible, the $K_{\mathrm{p}}$ values can be refined via fitting approach using observed arterial and arm vein concentrations simultaneously.

The critical importance of the choice of the sampling site in PBPK models is further emphasized by the impact on model verification, parameter optimization, and model extrapolation. A common practice of top-down PBPK model development is to optimize experimentally undetermined parameters until the observed data are captured by the simulation. Therefore, the choice of simulation output is critical. Choosing a PBPK model sampling site that is different from the actual site used to collect observed PK data is likely to specifically lead to erroneous parameter optimization and rejection of true model parameters due to the inherent differences between arterial and arm vein concentrations. This observation is particularly important for parameters that are poorly experimentally characterized and therefore typically empirically optimized, such as the tissue-to-plasma partition coefficient and the absorption rate constant. This concept is illustrated in the simulations of fentanyl disposition after buccal administration using the fentanyl model verified for both arterial and arm vein sampling. When concentrations were sampled from the central venous compartment (right atrium) in the simulations but compared with the observed arm vein concentrations, the verified fentanyl model with the true parameters failed to meet model acceptance criteria. As a result, fentanyl absorption parameters were optimized erroneously to compensate for the inherent sampling sitedependent discrepancy. Importantly, these erroneously optimized model parameters mispredicted the impact of changes in formulation and absorption rate on $C_{\max }$ by 3 -fold, leading to incorrect conclusions of drug safety and efficacy.

A similar issue of misfitting or model misspecification was discussed previously regarding how arteriovenous differences affect the accurate derivation of PK-PD relationships and therefore the prediction of PD (Gumbleton et al., 1994; Jacobs and Nath, 1995; Tuk et al., 1997). Previous PK-PD communications regarding arteriovenous differences have mainly used empirically fitted compartmental models, and hence cannot be directly compared with mechanistic PBPK models. It has been proposed that coupling the PBPK model to the PK-PD model can provide a mechanistic prediction of therapeutic effect given the capacity of the PBPK modeling to simulate tissue drug concentrations where the drug effects occur (Kuepfer et al., 2016; Pichardo-Almarza and Diaz-Zuccarini, 2016; Boger and Fridén, 2019). Since PK-PD models often assess the time course of pharmacological effect and how the onset of effect relates to plasma concentrations during the initial distribution phase, strong scrutiny to correct sampling site is necessitated during model development.

Finally, our study extends the PBPK model application to explore the unstudied scenario of arteriovenous concentration differences for metabolites after intravenous and oral administration. Using simulations, we discovered an atypical metabolite profile resulting from central venous compartment (right atrium) sampling showing a spike during the early distribution phase of the parent drug. This unique phenomenon is caused by initial high concentrations of parent drug during its distribution phase and rapid appearance of metabolite in the central venous compartment (right atrium) due to unrestricted passive diffusion across the sinusoidal membrane for metabolites formed in the liver. This atypical metabolite spike observed from the simulated central venous metabolite profile was hardly observed from the simulated peripheral arm vein metabolite profile due to tissue distribution and dilution at the sampling site. The simulated peripheral arm vein metabolite profiles shown here agree with commonly observed metabolite profiles from clinical studies.

In conclusion, this study shows that the developed PBPK model with a peripheral sampling compartment can successfully recapitulate arteriovenous drug concentration differences and it is critically important to match the PBPK model sampling site with the experimental sampling site used in clinical studies, especially for drugs with large volumes of distribution or high clearances. We also show that arteriovenous differences can be considerable after oral dosing and for drug metabolites. The use of a discrepant sampling site may lead to biased model evaluation, erroneous parameter optimization, and clinically significant mispredictions of plasma concentration-time curves. As PBPK-PD models are being developed, attention needs to be paid to assessing arteriovenous differences and using comparable sampling sites between simulations and observations.

\section{Authorship Contributions}

Participated in research design: Huang, Isoherranen.

Conducted experiments: Huang.

Performed data analysis: Huang, Isoherranen.

Wrote or contributed to the writing of the manuscript: Huang, Isoherranen.

\section{References}

Boger E and Fridén M (2019) Physiologically based pharmacokinetic/pharmacodynamic modeling accurately predicts the better bronchodilatory effect of inhaled versus oral salbutamol dosage forms. J Aerosol Med Pulm Drug Deliv 32:1-12.

Britz H, Hanke N, Volz AK, Spigset O, Schwab M, Eissing T, Wendl T, Frechen S, and Lehr T (2019) Physiologically-based pharmacokinetic models for CYP1A2 drug-drug interaction prediction: a modeling network of fluvoxamine, theophylline, caffeine, rifampicin, and midazolam. CPT Pharmacometrics Syst Pharmacol 8:296-307.

Chiou WL (1989) The phenomenon and rationale of marked dependence of drug concentration on blood sampling site. Implications in pharmacokinetics, pharmacodynamics, toxicology and therapeutics (part I). Clin Pharmacokinet 17:175-199.

Cooper KE, Edholm OG, and Mottram RF (1955) The blood flow in skin and muscle of the human forearm. J Physiol 128:258-267.

Darwish M, Kirby M, Robertson P Jr, Hellriegel E, and Jiang JG (2006a) Comparison of equivalent doses of fentanyl buccal tablets and arteriovenous differences in fentanyl pharmacokinetics. Clin Pharmacokinet 45:843-850.

Darwish M, Kirby M, Robertson P Jr, Tracewell W, and Xie F (2010) Dose proportionality of fentanyl buccal tablet in doses ranging from 600 to 1300 microg in healthy adult subjects: a randomized, open-label, four-period, crossover, singlecentre study. Clin Drug Investig 30:365-373.

Darwish M, Tempero K, Kirby M, and Thompson J (2006b) Relative bioavailability of the fentanyl effervescent buccal tablet (FEBT) $1,080 \mathrm{pg}$ versus oral transmucosal fentanyl citrate $1,600 \mathrm{pg}$ and dose proportionality of FEBT 270 to 1,300 microg: a single-dose, randomized, open-label, three-period study in healthy adult volunteers. Clin Ther 28:715-724.

Edwards SR and Mather LE (2001) Tissue uptake of ketamine and norketamine enantiomers in the rat: indirect evidence for extrahepatic metabolic inversion. Life Sci 69:2051-2066.

Elia M and Kurpad A (1993) What is the blood flow to resting human muscle? Clin Sci (Lond) 84:559-563.

Ericsson H, Bredberg U, Eriksson U, Jolin-Mellgård A, Nordlander M, and Regårdh CG (2000) Pharmacokinetics and arteriovenous differences in clevidipine concentration following a short- and a long-term intravenous infusion in healthy volunteers. Anesthesiology 92:993-1001.

Goodman LS, Gilman A, Brunton LL, Lazo JS, and Parker KL (2006) Goodman \& Gilman's The Pharmacological Basis of Therapeutics, 11th ed, McGraw-Hill, New York.

Gourlay SG and Benowitz NL (1997) Arteriovenous differences in plasma concentration of nicotine and catecholamines and related cardiovascular effects after smoking, nicotine nasal spray, and intravenous nicotine. Clin Pharmacol Ther 62:453-463.

Grimstein M, Yang Y, Zhang X, Grillo J, Huang S-M, Zineh I, and Wang Y (2019) Physiologically based pharmacokinetic modeling in regulatory science: an update from the U.S. Food and Drug Administration's Office of Clinical Pharmacology. J Pharm Sci 108:21-25.

Gumbleton M, Oie S, and Verotta D (1994) Pharmacokinetic-pharmacodynamic (PK $\mathrm{PD}$ ) modelling in non-steady-state studies and arterio-venous drug concentration differences. Br J Clin Pharmacol 38:389-400. 
Huang SM, Abernethy DR, Wang Y, Zhao P, and Zineh I (2013) The utility of modeling and simulation in drug development and regulatory review. J Pharm Sci 102: 2912-2923.

Huang W and Isoherranen N (2018) Development of a dynamic physiologically based mechanistic kidney model to predict renal clearance. CPT Pharmacometrics Syst Pharmacol 7:593-602.

Huang W, Nakano M, Sager J, Ragueneau-Majlessi I, and Isoherranen N (2017) Physiologically based pharmacokinetic model of the CYP2D6 probe atomoxetine: extrapolation to special populations and drug-drug interactions. Drug Metab Dispos 45:1156-1165.

Isohanni MH, Ahonen J, Neuvonen PJ, and Olkkola KT (2005) Effect of ciprofloxin on the pharmacokinetics of intravenous lidocaine. Eur J Anaesthesiol 22:795-799.

Jacobs JR and Nath PA (1995) Compartment model to describe peripheral arterialvenous drug concentration gradients with drug elimination from the venous sampling compartment. J Pharm Sci 84:370-375.

Jamei M (2016) Recent advances in development and application of physiologicallybased pharmacokinetic (PBPK) models: a transition from academic curiosity to regulatory acceptance. Curr Pharmacol Rep 2:161-169.

Jones H and Rowland-Yeo K (2013) Basic concepts in physiologically based pharmacokinetic modeling in drug discovery and development. CPT Pharmacometrics Syst Pharmacol 2:e63.

Jones HM, Dickins M, Youdim K, Gosset JR, Attkins NJ, Hay TL, Gurrell IK, Logan YR, Bungay PJ, Jones BC, et al. (2012) Application of PBPK modelling in drug discovery and development at Pfizer. Xenobiotica 42:94-106.

Jones HM, Mayawala K, and Poulin P (2013) Dose selection based on physiologically based pharmacokinetic (PBPK) approaches. AAPS J 15:377-387.

Jones HM, Parrott N, Jorga K, and Lavé T (2006) A novel strategy for physiologically based predictions of human pharmacokinetics. Clin Pharmacokinet 45:511-542.

Kaur N, Narang A, and Bansal AK (2018) Use of biorelevant dissolution and PBPK modeling to predict oral drug absorption. Eur J Pharm Biopharm 129:222-246.

Khalil F and Läer S (2011) Physiologically based pharmacokinetic modeling: methodology, applications, and limitations with a focus on its role in pediatric drug development. J Biomed Biotechnol 2011:907461.

Kuepfer L, Niederalt C, Wendl T, Schlender JF, Willmann S, Lippert J, Block M, Eissing T, and Teutonico D (2016) Applied concepts in PBPK modeling: how to build a PBPK/PD model. CPT Pharmacometrics Syst Pharmacol 5:516-531.

Levitt DG (2004) Physiologically based pharmacokinetic modeling of arterial antecubital vein concentration difference. BMC Clin Pharmacol 4:2.

Luzon E, Blake K, Cole S, Nordmark A, Versantvoort C, and Berglund EG (2017) Physiologically based pharmacokinetic modeling in regulatory decision-making at the European Medicines Agency. Clin Pharmacol Ther 102:98-105.

Macleod DB, Habib AS, Ikeda K, Spyker DA, Cassella JV, Ho KY, and Gan TJ (2012) Inhaled fentanyl aerosol in healthy volunteers: pharmacokinetics and pharmacodynamics. Anesth Analg 115:1071-1077.

Miller NA, Reddy MB, Heikkinen AT, Lukacova V, and Parrott N (2019) Physiologically based pharmacokinetic modelling for first-in-human predictions: an updated model building strategy illustrated with challenging industry case studies. Clin Pharmacokinet 58:727-746.

Min JS and Bae SK (2017) Prediction of drug-drug interaction potential using physiologically based pharmacokinetic modeling. Arch Pharm Res 40:1356-1379.

Moksnes K, Fredheim OM, Klepstad P, Kaasa S, Angelsen A, Nilsen T, and Dale O (2008) Early pharmacokinetics of nasal fentanyl: is there a significant arteriovenous difference? Eur J Clin Pharmacol 64:497-502.

Musther H, Gill KL, Chetty M, Rostami-Hodjegan A, Rowland M, and Jamei M (2015) Are physiologically based pharmacokinetic models reporting the right $\mathrm{C}(\max )$ ? Central venous versus peripheral sampling site. AAPS J 17:1268-1279.
Offman E and Edginton AN (2015) A PBPK workflow for first-in-human dose selection of a subcutaneously administered pegylated peptide. J Pharmacokinet Pharmacodyn 42:135-150.

Olofsen E, Mooren R, van Dorp E, Aarts L, Smith T, den Hartigh J, Dahan A, and Sarton E (2010) Arterial and venous pharmacokinetics of morphine-6-glucuronide and impact of sample site on pharmacodynamic parameter estimates. Anesth Analg 111:626-632.

Persson J, Hasselström J, Maurset A, Oye I, Svensson JO, Almqvist O, Scheinin H, Gustafsson LL, and Almqvist O (2002) Pharmacokinetics and non-analgesic effects of S- and R-ketamines in healthy volunteers with normal and reduced metabolic capacity. Eur J Clin Pharmacol 57:869-875.

Pichardo-Almarza C and Diaz-Zuccarini V (2016) From PK/PD to QSP: understanding the dynamic effect of cholesterol-lowering drugs on atherosclerosis progression and stratified medicine. Curr Pharm Des 22:6903-6910.

Pitsiu M, Gries JM, Benowitz N, Gourlay SG, and Verotta D (2002) Modeling nicotine arterial-venous differences to predict arterial concentrations and input based on venous measurements: application to smokeless tobacco and nicotine gum. J Pharmacokinet Pharmacodyn 29:383-402.

Rentsch KM, Kullak-Ublick GA, Reichel C, Meier PJ, and Fattinger K (2001) Arteria and venous pharmacokinetics of intravenous heroin in subjects who are addicted to narcotics. Clin Pharmacol Ther 70:237-246.

Rodgers T, Leahy D, and Rowland M (2005) Physiologically based pharmacokinetic modeling 1: predicting the tissue distribution of moderate-to-strong bases $J$ Pharm Sci 94:1259-1276.

Rodgers T and Rowland M (2007) Mechanistic approaches to volume of distribution predictions: understanding the processes. Pharm Res 24:918-933.

Rowland M, Peck C, and Tucker G (2011a) Physiologically-based pharmacokinetics in drug development and regulatory science. Annu Rev Pharmacol Toxicol 51:45-73

Rowland Yeo K, Aarabi M, Jamei M, and Rostami-Hodjegan A (2011b) Modeling and predicting drug pharmacokinetics in patients with renal impairment. Expert Rev Clin Pharmacol 4:261-274.

Sager JE, Yu J, Ragueneau-Majlessi I, and Isoherranen N (2015) Physiologically based pharmacokinetic (PBPK) modeling and simulation approaches: a systematic review of published models, applications, and model verification. Drug Metab Dispos 43:1823-1837.

Shebley M, Sandhu P, Emami Riedmaier A, Jamei M, Narayanan R, Patel A, Peters SA, Reddy VP, Zheng M, de Zwart L, et al. (2018) Physiologically based pharmacokinetic model qualification and reporting procedures for regulatory submissions: a consortium perspective. Clin Pharmacol Ther 104:88-110.

Tucker GT and Boas RA (1971) Pharmacokinetic aspects of intravenous regional anesthesia. Anesthesiology 34:538-549.

Tuk B, Danhof M, and Mandema JW (1997) The impact of arteriovenous concentration differences on pharmacodynamic parameter estimates. $J$ Pharmacokinet Biopharm 25:39-62.

U.S. Food and Drug Administration (2011) Fentanyl (FENTORA): US prescribing information.

Ziesenitz VC, König SK, Mahlke NS, Skopp G, Haefeli WE, and Mikus G (2015) Pharmacokinetic interaction of intravenous fentanyl with ketoconazole. $J$ Clin Pharmacol 55:708-717.

Address correspondence to: Nina Isoherranen, Department of Pharmaceutics, School of Pharmacy, University of Washington, Health Science Building, Room H-272N, Box 357610, Seattle, WA 98195-7610. E-mail: ni2@ u.washington.edu 